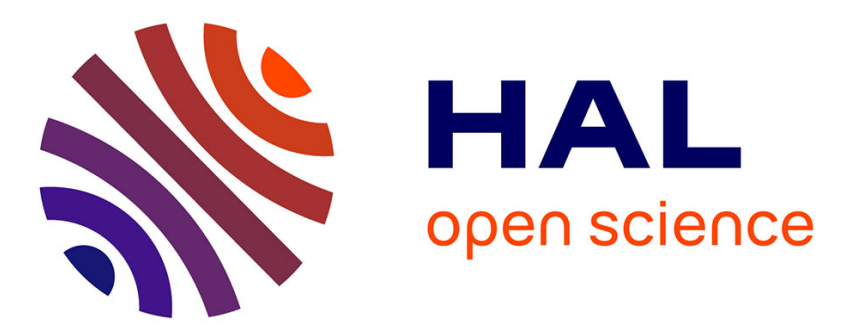

\title{
Numerical identification of parameters for a model of sedimentation processes
}

Mauricio Sepulveda, Francois James, Anibal Coronel

\section{To cite this version:}

Mauricio Sepulveda, Francois James, Anibal Coronel. Numerical identification of parameters for a model of sedimentation processes. Inverse Problems, 2003, 19, pp.951-972. 10.1088/02665611/19/4/311. hal-00079236

\section{HAL Id: hal-00079236 https://hal.science/hal-00079236}

Submitted on 9 Jun 2006

HAL is a multi-disciplinary open access archive for the deposit and dissemination of scientific research documents, whether they are published or not. The documents may come from teaching and research institutions in France or abroad, or from public or private research centers.
L'archive ouverte pluridisciplinaire HAL, est destinée au dépôt et à la diffusion de documents scientifiques de niveau recherche, publiés ou non, émanant des établissements d'enseignement et de recherche français ou étrangers, des laboratoires publics ou privés. 


\title{
Numerical identification of parameters for a model of sedimentation processes
}

\author{
Aníbal Coronel†, François Jamesł and Mauricio Sepúlveda† \\ † GI $\mathbf{I}^{2} \mathbf{M A}$, Departamento de Ingeniería Matemática, Universidad de Concepción, \\ Casilla 160-C,Concepción, Chile \\ ‡ Mathématiques, Applications et Physique Mathématique d'Orléans, UMR CNRS \\ 6628, UFR Sciences, Université d'Orléans, B.P. 6759, F-45067 Orléans Cedex 2, \\ France. \\ E-mail: acoronel@ing-mat.udec.cl, james@labomath.univ-orleans.fr and \\ mauricio@ing-mat.udec.cl
}

\begin{abstract}
.
In this paper we present the identification of parameters in the flux and diffusion functions for a quasilinear strongly degenerate parabolic equation which models the physical phenomenon of flocculated sedimentation. We formulate the identification problem as a minimization of a suitable cost function and we derive its formal gradient by means of adjoint equation which is a backward linear degenerate parabolic equation with discontinuous coefficients. For the numerical approach, we start with the discrete Lagrangian formulation and assuming that the direct problem is discretized by the Engquist-Osher scheme obtain a discrete adjoint state associated to this scheme. The conjugate gradient method permits to find numerically the physical parameters. In particular, it allows to identify as well the critical concentration level at which solid flocs begin to touch each other and determines the change of parabolic to hyperbolic behavior in the model equation.
\end{abstract}

\section{Introduction}

Batch sedimentation is a classical procedure to separate flocculated suspension into a concentrated sediment of practical interest, and a clear fluid. It is used, for example in metallurgy, alimentation industry. The experimental setting consists of a vertical column, with a surface feeding at the top, and a discharge surface at the bottom. Under the influence of gravity, the suspension separates into a clear fluid, and a compressible sediment, which is collected at the bottom of the column. We refer to $[13,3,4,6]$ for more complete descriptions and details.

Under several constitutive and simplifying assumptions, it turns out that this mixture of two continuous media (fluid and solid flocs) can be described by a single model equation, namely a partial differential equation of mixed type, hyperbolic and parabolic. The unknown is the volumetric solid concentration $\phi$, which is a function of the time $t$ and the height in the column $z$. 
Actually, the mixed type nature of the equation results from the different behaviour of the solid flocs on the one hand, the fluid on the other. The former indeed lies in a compression region, where the solid effective stress $\sigma_{e}$ is not constant, while in the fluid zone, it can be taken equal to a constant. Therefore, in the solid zone (sediment), the equation will be of parabolic type, in the fluid zone, it will be hyperbolic.

More precisely, it can be assumed that $\sigma_{e}$ is a nonlinear function of $\phi$ alone, with the following shape:

$$
\sigma_{e}^{\prime}(\phi)= \begin{cases}=0 & \text { for } \phi \leq \phi_{c} \\ >0 & \text { for } \phi>\phi_{c}\end{cases}
$$

The concentration $\phi_{c}$ is the so-called critical concentration, above which the flocs get into contact with each other and form a network. The location of the interface $\phi=\phi_{c}$ is a specific problem in direct simulations.

Another constitutive relation can be obtained through Kynch's theory of kinematic sedimentation. It gives the velocity of the mixture as a function of the concentration $\phi$,

$$
f(\phi, t)=q(\phi, t)+f_{b k}(\phi)
$$

Here $q$ is the volume average velocity of the mixture, $q=v_{s} \phi+v_{f}(1-\phi)\left(v_{s}\right.$ and $v_{f}$ are the solid and fluid velocities), and $f_{b k}$ the so-called batch flux density function. Kynch's theory gives a precise expression for this function.

We are interested here in the inverse problem which consists in identifying the constitutive laws $f_{b k}$ and $\sigma_{e}$ from experimental data. This kind of problem is in general impossible to solve in its full generality, that is considering $f_{b k}$ and $\sigma_{e}$ as general functions. Therefore we shall consider more precise constitutive assumptions, which give explicit expressions for both functions, depending on a finite number of parameters. Among these we emphasize the value of the critical concentration $\phi_{c}$, which is of great practical importance, and very difficult to access from experimental data. We mention here that there are several experimental methods to obtain the involved parameters, see $[11,1]$ for an overview. They employ a set of data of local solid concentration and local permeability to the effective solid stress to obtain approximate correlation formulas and then by algebraic manipulation of this correlations determinate some empirical representation of $f_{b k}$ and $\sigma_{e}$. In contrast to our approach, we need only experimental concentration data and we compute the optimal $f_{b k}$ and $\sigma_{e}$.

There is a large list of authors who propose analytical and numerical methods for inverse problems in evolution partial differential equations. For example, parameter identification methods for parabolic PDEs can be found in $[14,15,24,29,31]$ and references therein. Recently, Yamamoto and Zou reconstruct in [33] the radiative coefficient and the initial data for a linear parabolic equations using a piecewise linear finite element method for the discretization and a nonlinear gradient multigrid method for accelerating the reconstruction process. Several difficulties arise in the case specific we consider, since we want to reconstruct nonlinear coefficients, but such a strategy is likely useful to improve the results. Moreover, the hyperbolic degeneracy gives rise to 
shocks in the concentration profiles. The existence and uniqueness for weak solutions to the direct problem rely on specific entropy conditions which has physical relevancy for the model and must be considered owing to the nonlinearity of the flux and degeneracy of the diffusion, see $[3,4,9]$.

The existence of solutions for the inverse problem is a consequence of the continuous dependence of the entropy solutions with respect to the flux and the diffusion (see Theorem 2 below and [19, 21]). Its uniqueness cannot be ensured because of the hyperbolic behaviour, see $[25,26]$. Therefore we shall rewrite the identification problem by adapting the technique developed by James and Sepúlveda, in [25, 26, 27], which is numerically tested as an efficient method to reconstruct the flux of a particular hyperbolic system. The idea is to write the inverse problem as an optimization problem for an appropriate cost function and then to apply the classical conjugate gradient method.

The continuous gradient stems from an adjoint state to the model consisting of a backward linear degenerate convection-diffusion equation with discontinuous coefficients and boundaryconditions. As in the purely hyperbolic case, the adjoint equation is illposed in uniqueness. We obtain the discrete gradient by computing the exact gradient of the discrete formulation of the optimization problem. This has become a classical technique, instead of computing the discretization of the formal gradient, because identification problems are generally badly conditioned or ill-posed, see [17, 27]. All the computations are based upon the explicit (first and second order), semi-implicit and implicit Engquist-Osher (or generalized upwind scheme) for the numerical computation of the solution of the sedimentation model, see Bürger et al [9]. In each case, the adjoint scheme and the discrete gradient are provided.

The remainder of this paper is organized as follows. In section 2 we provide the formulation of the direct and inverse problems. In section 3 we analyze the question of the well posedness. In section 4 we present the formal calculus of the gradient. In section 5 we introduce the numerical schemes for the identification of the parameters and we present some numerical results.

\section{Statement of the problem}

\subsection{The direct problem}

Summarizing the results given in $[2,3,4,6]$ for the mathematical model of the sedimentation processes, we have the following IBVP

$$
\begin{array}{rlrl}
\frac{\partial \phi}{\partial t}+\frac{\partial}{\partial z}\left(q(t) \phi+f_{b k}(\phi)\right) & =-\frac{\partial}{\partial z}\left(f_{b k}(\phi) \frac{\sigma_{e}^{\prime}(\phi)}{\Delta \rho g \phi} \frac{\partial \phi}{\partial z}\right),(z, t) \in Q_{T},(1) \\
& =\phi_{0}(z), & z \in[0, L], & \\
\phi(z, 0) & =\phi_{2}(t), & t \in[0, T], & \\
\phi(L, t) & =0, & t \in[0, T] \\
\left.f_{b k}(\phi)\left(1+\frac{\sigma^{\prime}(\phi)}{\Delta \rho g \phi} \frac{\partial \phi}{\partial z}\right)\right|_{z=0} & =0
\end{array}
$$


where $\phi$ is the non-negative unknown function, $Q_{T}=[0, L] \times[0, T], \Delta \rho$ and $g$ are positive constants, $q, f_{b k}, \sigma_{e}, \phi_{0}$ and $\phi_{2}$ are given functions with the next supposed behavior

- $q$ is a non-positive Lipschitz function, this is

$$
q \in \operatorname{Lip}([0, T]) \text { and } q(t) \leq 0, \forall t \in[0, T] .
$$

- $f_{b k}(\phi)$ is a smooth function such that

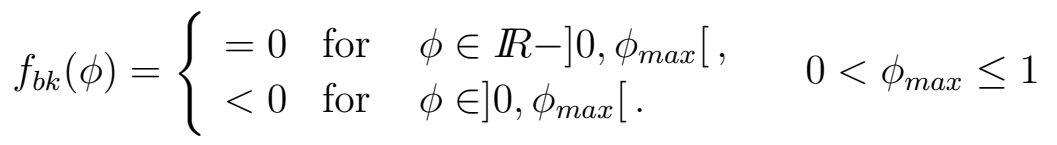

- $\sigma_{e}(\phi)$ is a $C^{3}$ function, constant for $\phi<\phi_{c}$, with monotonic increasing for $\phi>\phi_{c}$ where $\left.\phi_{c} \in\right] 0, \phi_{\max }$ [ is a constant, and its first derivative, $\sigma_{e}^{\prime}$, satisfies

$$
\sigma_{e}^{\prime}(\phi)=\left\{\begin{array}{lll}
=0 & \text { for } \quad \phi \leq \phi_{c} \\
>0 & \text { for } \quad \phi>\phi_{c}
\end{array}\right.
$$

- $\phi_{0}$ and $\phi_{2}$ are piecewise continuous functions such that

$$
0 \leq \phi_{0}(z), \phi_{2}(t) \leq \phi_{\max }, \quad z \in[0, L], \quad t \in[0, T]
$$

with $\phi_{2}$ changing its monotonicity behavior a finite number of times.

In the setting of sedimentation theory $\phi(z, t)$ denotes the volumetric solid concentration at height $z$ in time $t, q$ the volume-averaged velocity of a suspension, $f_{b k}$ the Kynch batch flux density function, $\phi_{\max }$ is the maximum concentration value, $\sigma_{e}$ the effective solid stress, $L$ the height of thickener feeding level, $T$ the total time for the process, $\Delta \rho$ the difference of solid and fluid mass densities, $g$ the acceleration of gravity and $\phi_{c}$ is a critical concentration value or gel point, see $[2,13,5,6]$ for specific details.

The flux density function (or shortly flux) and the diffusion coefficient (or shortly diffusion) associated to equation (1) are defined by

$$
f(\phi, t)=q(t) \phi+f_{b k}(\phi) \quad \text { and } \quad a(\phi)=-\frac{f_{b k}(\phi) \sigma_{e}^{\prime}(\phi)}{\Delta \rho g \phi},
$$

respectively. Moreover we define the integrated diffusion coefficient $A$ by

$$
A(\phi)=\int_{0}^{\phi} a(s) d s .
$$

Due to (6), (7) and (9), (1) is a second order parabolic partial differential equation for $\phi \in] \phi_{c}, \phi_{\max }[$, a nonlinear hyperbolic conservation law for $\phi \in] 0, \phi_{c}[$ and a linear advection equation for $\phi \in \mathbb{R}-\left[0, \phi_{\max }\right]$. In brief, the IBVP is referenced as a quasilinear strongly degenerate parabolic equation. We remark that it is sufficient to consider the degeneracy on $\left[0, \phi_{c}\right] \cup\left\{\phi_{\max }\right\}$ because for $\phi$ solution of the IBVP we have that $\phi \in\left[0, \phi_{\max }\right]$ almost everywhere, see [4]. 


\subsection{The inverse problem}

Experimental results obtained in industrial and laboratory processes for flocculated sedimentation suggest to consider in the model (1)-(9), a dependence of finite number of parameters for the functions $f_{b k}$ and $\sigma_{e}$, see $[2,13,5]$ and [6]. The determination of this parameters implies to solve an "Identification Problem", IP. We can formulate it, in general way, as follows

IP Given an observation data $\phi^{\text {obs }}(z)$, at time $T$, and the functions $q(t), \phi(z, 0), \phi(L, t)$, explicitly, and $\phi(0, t)$, implicitly, satisfying (5), (2), (3) and (4) respectively, find the flux $f$ and the diffusion a with $f_{b k}$ and $\sigma_{e}$ satisfying (6) and (7) such that the weak solution $\phi(z, T)$, in time $T$, of the IBVP (1)-(7) is as close as to $\phi^{\text {obs }}(z)$ in some suitable norm.

We can write the IP, see $[25,26]$ and $[27]$, as the optimization of a cost function $J$

$$
\min _{f, a} J(\phi(\cdot, T))
$$

under the constraint for $\phi$ to satisfy weakly the IBVP (1)-(4), for some $f$ and $a$. A natural example of cost function $J$ is

$$
J(\phi)=\frac{1}{2} \int_{0}^{L}\left|\phi(z, T)-\phi^{o b s}(z)\right|^{2} d z,
$$

for other examples see [26].

We particularize the general situation by consider the parametric dependent analytic form of the flux and the diffusion. In this work, we seek $f_{b k}$ by the usual formula of Richardson and Zaki [32], and $\sigma_{e}$ by a constitutive law, [9], i.e.

$$
\begin{aligned}
& f_{b k}(\phi)=u_{\infty} \phi\left(1-\frac{\phi}{\phi_{\max }}\right)^{C}, \\
& \sigma_{e}(\phi)= \begin{cases}\text { Cte. } & \text { for } \phi \leq \phi_{c}, \\
\alpha\left(\left(\phi / \phi_{c}\right)^{\beta}-1\right) & \text { for } \phi>\phi_{c},\end{cases}
\end{aligned}
$$

where $u_{\infty}$ is the flow velocity of a singular particle in an unbounded medium and $\left.C>1, \alpha>0, \beta>1, \phi_{c} \in\right] 0, \phi_{\max }[$ are parameters(see $[13,23,11]$ for another examples). Thus, in this particular case, if we denote by e the parameters to find, the problem (11) can be formulated in an equivalent way by

$$
\min _{\mathbf{e}} J(\phi \mathbf{e}(\cdot, T))
$$

where $\phi_{\mathbf{e}}$ is a weak solution of IBVP (1)-(4) with $f_{b k}$ and $\sigma_{e}$ given by (13)-(14), respectively.

The nonlinearity and degeneracy implies that solutions of the IBVP may become discontinuous in finite time. Thus, we need to interpret the IBVP in a weak way. In addition, it is well known that the IBVP is ill-posed in the classical weak sense because there is no uniqueness. In order to have a well-posed problem we must consider an additional condition or entropy condition, see [3, 4, 10]. 


\section{Theoretical analysis of the IBVP and the IP}

\subsection{The direct problem}

We adopt in this work the definition of weak entropy solution introduced by Bürger et al. in [10].

Definition 1 Let $\phi \in L^{\infty}\left(Q_{T}\right) \cap B V\left(Q_{T}\right)$. Then $\phi$ is an entropy solution of the IBVP if the following four conditions are valid

(i) $\partial_{z} A(\phi) \in L^{2}\left(Q_{T}\right)$

(ii) For all $k \in \mathbb{R}$ and for all $\left.\left.\varphi \in C^{\infty}(] 0,1\right] \times[0, T]\right)$ such that $\varphi \geq 0$ and $\operatorname{supp} \varphi \subset] 0,1] \times] 0, T[$ the entropy inequality

$$
\begin{aligned}
& \iint_{Q_{T}}\left\{|\phi-k| \partial_{t} \varphi+\operatorname{sgn}(\phi-k)\left(f(\phi, t)-f(k, t)-\partial_{z} A(\phi)\right) \partial_{z} \varphi\right\} d z d t \\
& +\int_{0}^{T}\left\{-\operatorname{sgn}\left(\phi_{2}(t)-k\right)\left[f\left(\gamma_{1} \phi, t\right)-f(k, t)-\gamma_{1} \partial_{z} A(\phi)\right] \varphi(1, t)\right. \\
& \left.\quad+\left[\operatorname{sgn}\left(\gamma_{1} \phi-k\right)-\operatorname{sgn}\left(\phi_{2}(t)-k\right)\right]\left[A\left(\gamma_{1} \phi\right)-A(k)\right] \partial_{z} \varphi(1, t)\right\} d t \geq 0
\end{aligned}
$$

is satisfied.

(iii) For almost all $t \in] 0, T[$

$$
\gamma_{0}\left(f_{b k}(\phi)-\partial_{z} A(\phi)\right)=0
$$

(iv) For almost all $z \in[0, L]$

$$
\lim _{t \rightarrow 0} \phi(z, t)=\phi_{0}(z)
$$

The notations $\gamma_{0}$ and $\gamma_{1}$ denote the traces in $B V\left(Q_{T}\right)$ at $x=0$ and $x=1$, respectively. For a precise definition see [4]. The item (i) is a technical regularity condition, (ii) is the entropy condition meanwhile that (iii) and (iv) are the weak formulation for the boundary condition (4) and the initial condition (2), respectively.

The definition 1 implies that the direct problem is well-posed, it was proved in [10], see also [4]. In that works, following the Kružkov [30] and Carrillo [16] ideas they establish several properties for the entropy solution of the direct problem. In particular they, provided the hypothesis (5)-(9), proved the following theorem

Theorem 1 There is almost one entropy solution $\phi \in B V\left(Q_{T}\right)$ for the IBVP (1)-(4).

\subsection{Existence of solutions to the Inverse Problem}

In this section we provide a sufficient condition for existence of at least one solution for our IP. The existence result is a consequence of the continuous dependence of the entropy solution with respect to the flux and diffusion. The non-uniqueness is a consequence of the degeneracy and the hyperbolic behavior. 
The continuous dependence for a Cauchy Problem with spatially dependent flux was studied in $[28,21]$. Their ideas, inspired by the works of Carrillo [16] and Cockburn and Gipenberg [19], can be extended to our IBVP with time dependent flux. First, we need the following lemma.

Lemma 1 Assume that $A^{\prime}>0$, then for any $\varphi \geq 0$ in $C_{0}^{\infty}\left(Q_{T}\right)$ and $k \in \mathbb{R}$ we have

$$
\begin{gathered}
\iint_{Q_{T}}\left\{|\phi-k| \partial_{t} \varphi+\operatorname{sgn}(\phi-k)\left(f(\phi, t)-f(k, t)-\partial_{z} A(\phi)\right) \partial_{z} \varphi\right\} d t d z \\
=\lim _{\epsilon \rightarrow 0} \iint_{Q_{T}} A^{\prime}(\phi)\left(\partial_{z} \phi\right)^{2} \operatorname{sgn}_{\epsilon}^{\prime}(\phi-k) \varphi d t d z
\end{gathered}
$$

where $\phi$ is an entropy solution of the IBVP and

$$
\operatorname{sgn}_{\epsilon}(r)=\left\{\begin{array}{rl}
-1 & r<-\epsilon \\
r / \epsilon & -\epsilon \leq r \leq \epsilon \\
1 & r>\epsilon
\end{array}\right.
$$

Proof. Let $\psi_{\epsilon}(z)=-\operatorname{sgn}_{\epsilon}\left(A^{-1}(z)-k\right)$ and $A_{\psi_{\epsilon}}(\phi)=\int_{k}^{\phi} \psi_{\epsilon}(A(r)) d r$. Then, by a "weak chain rule" (see $[16,10])$ we have

$$
-\int_{0}^{T}<\partial_{t} \phi,-\operatorname{sgn}_{\epsilon}(\phi-k) \varphi>d t=\iint_{Q_{T}} A_{\psi_{\epsilon}}(\phi) \partial_{t} \varphi d t d z,
$$

where $<\cdot, \cdot>$ denotes the usual pairing between $H^{-1}(] 0, L[)$ and $H_{0}^{1}(] 0, L[)$.

On the other hand, by definition 1 , we follow that

$$
\begin{aligned}
-\int_{0}^{T}<\partial_{t} \phi, \operatorname{sgn}_{\epsilon}(\phi-k) \varphi>d t+ \\
\qquad \int_{Q_{T}}\left[f(\phi, t)-f(k, t)-\partial_{z} A(\phi)\right] \partial_{z}\left(\operatorname{sgn}_{\epsilon}(\phi-k) \varphi\right) d t d z=0 .
\end{aligned}
$$

Combining (16) with (17) and passing to the limit when $\epsilon \rightarrow 0$, we obtain (15).

Theorem 2 Let $u$ and $v$ be entropy solutions of the following IBVP's

$$
\begin{aligned}
& \frac{\partial u}{\partial t}+\frac{\partial}{\partial z}\left(q_{1}(t) u+f_{b k_{1}}(u)\right)=\frac{\partial^{2} A(u)}{\partial z^{2}} \\
& u(z, 0)=u_{0}(z), \quad u(L, t)=u_{L}(t), \quad f_{b k_{1}}(\phi)-\left.A(u) \frac{\partial u}{\partial z}\right|_{z=0}=0
\end{aligned}
$$

and

$$
\begin{aligned}
& \frac{\partial v}{\partial t}+\frac{\partial}{\partial z}\left(q_{2}(t) v+f_{b k_{2}}(v)\right)=\frac{\partial^{2} B(v)}{\partial z^{2}} \\
& v(z, 0)=v_{0}(z), \quad v(L, t)=v_{L}(t), \quad f_{b k_{2}}(v)-\left.B(v) \frac{\partial v}{\partial z}\right|_{z=0}=0
\end{aligned}
$$

respectively, where the assumptions (5)-(10) are fulfilled by each one of its coefficients, initial and boundary conditions. Then for almost all $t \in[0, T]$

$$
\begin{aligned}
\|u(\cdot, t)-v(\cdot, t)\|_{L^{1}([0, L])} \leq\left\|u_{0}-v_{0}\right\|_{L^{1}([0, L])}+t C\left\|q_{1}-q_{2}\right\|_{L i p([0, T])} \\
+t C\left\|f_{b k_{1}}-f_{b k_{2}}\right\|_{L i p\left(\left[0, u_{\max }\right]\right)}+\sqrt{t} C\|\sqrt{a}-\sqrt{b}\|_{L^{\infty}\left(\left[\phi_{c}, \phi_{\max }\right]\right)}
\end{aligned}
$$


where $C>0$ is a constant and $a(u)=A^{\prime}(u), b(v)=B^{\prime}(v)$.

Proof. It is sufficient to prove this theorem when the equations are uniformly parabolic since the degenerate parabolic case is a consequence of the convergence of the vanishing viscosity method, see $[4,10]$. The proof will be done by the generalized doubling of variables technique, see [16].

Let us introduce the test function $\varphi \in C_{0}^{\infty}\left(Q_{T} \times Q_{T}\right)$

$$
\varphi(z, t, y, s)=\left\{\int_{-\infty}^{t-\nu} \rho_{\theta}(s) d s-\int_{-\infty}^{t-\tau} \rho_{\theta}(s) d s\right\} \rho_{\eta}(z-y) \rho_{\delta}(t-s),
$$

where $\nu, \tau \in] 0, T\left[\right.$ are Lebesgue points of $\left.\|u(\cdot, t)-v(\cdot, t)\|_{L^{1}([0, L])}, \theta \in\right] 0, \min \{\nu, T-\tau\}[$, $\eta, \delta>0$ and $\rho_{\epsilon}(x)=(1 / \epsilon) \rho(x / \epsilon)$ for $\epsilon>0$ with $\rho \in C_{0}^{\infty}(\mathbb{R})$ such that $\rho$ is a even function, $\rho(r)=0$ for $|r|>1$ and $\int \rho(r) d r=1$.

We apply the lemma 1 twice. Firstly with $\varphi$ as a test function in $(z, t), \phi=u(z, t)$, $k=v(y, s)$ and integrate with respect to $(y, s) \in Q_{T}$. Then we apply the lemma with $\varphi$ as a test function in $(y, s), \phi=v(y, s)$ and $k=u(z, t)$ and integrate with respect to $(z, t) \in Q_{T}$. By summing up the results we obtain

$$
\begin{aligned}
-\iiint \int_{Q_{T} \times Q_{T}} & \left\{|u-v|\left(\partial_{t} \varphi+\partial_{s} \varphi\right)\right. \\
+ & \operatorname{sgn}(u-v)\left[(f(u, t)-f(v, t)) \partial_{z} \varphi-(g(v, s)-g(u, s)) \partial_{y} \varphi\right] \\
- & {\left.\left[\operatorname{sgn}(u-v) \partial_{z} A(u) \partial_{z} \varphi+\operatorname{sgn}(v-u) \partial_{y} B(v) \partial_{y} \varphi\right]\right\} d z d t d y d s } \\
= & -\lim _{\epsilon \rightarrow 0} \iiint \int_{Q_{T} \times Q_{T}}\left\{A^{\prime}(u)\left(\partial_{z} u\right)^{2}+B^{\prime}(v)\left(\partial_{y} v\right)^{2}\right\} \operatorname{sgn}_{\epsilon}^{\prime}(v-u) \varphi d z d t d y d s \\
\leq & -\lim _{\epsilon \rightarrow 0} \iiint \int_{Q_{T} \times Q_{T}} 2 \sqrt{A^{\prime}(u)} \sqrt{B^{\prime}(v)} \partial_{z} u \partial_{y} v \operatorname{sgn}_{\epsilon}^{\prime}(v-u) \varphi d z d t d y d s=S_{1}
\end{aligned}
$$

Now, by triangle inequality we get

$$
I_{1}+I_{2}+I_{3} \leq-\iiint \int_{Q_{T} \times Q_{T}}|u-v|\left(\partial_{t} \varphi+\partial_{s} \varphi\right) d z d t d y d s \leq S_{1}+S_{2}+S_{3}
$$

where

$$
\begin{aligned}
& I_{1}=-\iiint \int_{Q_{T} \times Q_{T}}|u(y, t)-v(y, t)|\left(\partial_{t} \varphi+\partial_{s} \varphi\right) d z d t d y d s \\
& I_{2}=-\iiint \int_{Q_{T} \times Q_{T}}|v(y, t)-v(y, s)|\left(\partial_{t} \varphi+\partial_{s} \varphi\right) d z d t d y d s \\
& I_{3}=-\iiint \int_{Q_{T} \times Q_{T}}|u(z, t)-u(y, t)|\left(\partial_{t} \varphi+\partial_{s} \varphi\right) d z d t d y d s \\
& S_{2}=\iiint \int_{Q_{T} \times Q_{T}} \operatorname{sgn}(u-v)\left[(f(u, t)-f(v, t)) \partial_{z} \varphi-(g(v, s)-g(u, s)) \partial_{y} \varphi\right] d z d t d y d s \\
& S_{3}=-\iiint \int_{Q_{T} \times Q_{T}}\left[\operatorname{sgn}(u-v) \partial_{z} A(u) \partial_{z} \varphi+\operatorname{sgn}(v-u) \partial_{y} B(v) \partial_{y} \varphi\right] d z d t d y d s .
\end{aligned}
$$

Taking into account that $\partial_{t} \varphi+\partial_{s} \varphi=\left[\rho_{\theta}(t-\nu)-\rho_{\theta}(t-\tau)\right] \rho_{\eta}(z-y) \rho_{\delta}(t-s)$ and 
$\partial_{z} \varphi+\partial_{y} \varphi=0$, we obtain that

$\lim _{\theta \rightarrow 0} I_{1}=\|u(\cdot, \tau)-v(\cdot, \tau)\|_{L^{1}(] 0, L[)}-\|u(\cdot, \nu)-v(\cdot, \nu)\|_{L^{1}(] 0, L[)}$,

$\lim _{\theta \rightarrow 0} I_{2}=0$

$\lim _{\theta \rightarrow 0} I_{3} \geq-2 \eta \sup _{t \in(\nu, \tau)}|u(\cdot, t)|_{B V(] 0, L[)}$,

$\lim _{\theta, \eta, \delta \rightarrow 0} S_{2} \leq\left(\left\|q_{1}-q_{2}\right\|_{L i p}+\left\|f_{b k 1}-f_{b k 2}\right\|_{L i p}\right) \sup _{t \in(\nu, \tau)}|u(\cdot, t)|_{B V(] 0, L[)}$

and

$\lim _{\theta \rightarrow 0} S_{1}+S_{3} \leq(\tau-\nu) \sup _{t \in(\nu, \tau)}|u(\cdot, t)|_{B V(] 0, L[)} \frac{2}{\eta}\left\|\sqrt{B^{\prime}}-\sqrt{A^{\prime}}\right\|_{L^{\infty}\left(\left[\phi_{c}, \phi_{\max }\right]\right)}^{2}$.

This estimates yields the result.

Corollary 1 Let $\mathcal{M}=\operatorname{Lip}([0, T]) \times \operatorname{Lip}\left(\left[0, u_{\max }\right]\right) \times L^{\infty}\left(\left[\phi_{c}, \phi_{\max }\right]\right)$. The mapping $\tilde{J}:\left(q, f_{b k}, a\right) \mapsto J\left(\phi_{q, f_{b k}, a}\right)$ defined from $\mathcal{M}$ to $\mathbb{R}_{+}$is continuous. Then, if $\left(q, f_{b k}, a\right) \in \mathcal{F}$, where $\mathcal{F}$ is a compact subset of $\mathcal{M}$, there exist at least one solution for the IP.

Because the square root is not a Lipschitz function it is interesting to note that if we consider the mapping $\hat{J}:\left(q, f_{b k}, \sqrt{a}\right) \mapsto J\left(\phi_{q, f_{b k}, a}\right)$ from $\mathcal{M}$ to $\mathbb{R}_{+}$is Lipschitz continuous, obtaining a strong result of continuity of the cost function respect to " $\sqrt{a}$ " than " $a$ ".

It is known that IP is ill-posed in uniqueness if we consider for instance the identification of the parameters of the flux an only shock observation, when $A=0$ (see [17] for more details). We hope to solve in practice this problem of uniqueness following the same idea of [17]: considering several experimental observations with rarefaction waves and limited number of real parameters to identify.

\section{Lagrangian formulation and formal calculus}

We define a Lagrangian for the problem (11) by setting

$$
\mathcal{L}(\phi, \psi ; f, a)=J(\phi)-E(\phi, \psi ; f, a),
$$

where $\psi$ is a smooth test function, $\phi$ is the state variable and

$$
\begin{aligned}
E(\phi, \psi ; f, a)= & -\int_{Q_{T}}\left(\phi \frac{\partial \psi}{\partial t}+f(\phi) \frac{\partial \psi}{\partial z}+A(\phi) \frac{\partial^{2} \psi}{\partial z^{2}}\right) \\
& +\int_{z=L}\left(\psi f\left(\phi_{2}\right)-\psi \frac{\partial A}{\partial z}\left(\phi_{2}\right)+A\left(\phi_{2}\right) \frac{\partial \psi}{\partial z}\right) \\
& -\int_{z=0}\left(\psi q(t) \phi+A(\phi) \frac{\partial \psi}{\partial z}\right)+\int_{t=T} \psi \phi-\int_{t=0} \psi \phi_{0} .
\end{aligned}
$$

When we formally take the derivative of $\mathcal{L}$ in the direction $\delta \phi$ we obtain

$$
\begin{aligned}
\left\langle\frac{\partial \mathcal{L}}{\partial \phi}, \delta \phi\right\rangle= & \int_{Q_{T}} \delta \phi\left(\frac{\partial \psi}{\partial t}+f^{\prime}(\phi) \frac{\partial \psi}{\partial z}+a(\phi) \frac{\partial^{2} \psi}{\partial z^{2}}\right) \\
& +\int_{z=0} \delta \phi\left(\psi q(t)+a(\phi) \frac{\partial \psi}{\partial z}\right)+\int_{t=T} \delta \phi\left(\phi-\phi^{o b s}-\psi\right),
\end{aligned}
$$


where we used the fact that $\phi_{0}, \phi_{2}$ and $\phi^{\text {obs }}$ are fixed. Now, we are interested in canceling $\partial \mathcal{L} / \partial \phi$, then $\psi$ should be solution of

$$
\begin{aligned}
\frac{\partial \psi}{\partial t}+\left(q(t)+f_{b k}^{\prime}(\phi)\right) \frac{\partial \psi}{\partial z} & =-a(\phi) \frac{\partial^{2} \psi}{\partial z^{2}}, & & (z, t) \in Q_{T}, \\
\psi(z, T) & =\phi(z, T)-\phi^{o b s}(z), & & z \in[0, L], \\
\psi q(t)-\left.a(\phi) \frac{\partial \psi}{\partial z}\right|_{z=0} & =0, & & t \in[0, T] .
\end{aligned}
$$

This problem is a backward boundary value problem for a linear parabolic degenerate equation with discontinuous coefficients. The end condition (19) depends on the cost function, in this case it corresponds to $J$. In more general case we have the relation $\int_{t=T} \delta \phi \psi=<\partial J / \partial \phi, \delta \phi>$, for all $\delta \phi$ smooth. No boundary condition is needed at $z=L$, for the case of batch sedimentation $q=\phi_{2}=0$, since the characteristics are entering the domain for the direct problem.

Let $\phi_{f, a}$ be solution of the IBVP. If $\phi_{f, a}$ is smooth, then we have $E\left(\phi_{f, a}, \psi ; f, a\right)=0$ for each $\psi$ smooth. In this way, $\mathcal{L}\left(\phi_{f, a}, \psi ; f, a\right)=J\left(\phi_{f, a}\right)=\tilde{J}(f, a)$. Thus

$$
\begin{aligned}
\nabla \tilde{J}(f, a) & =\left(\left\langle\frac{\partial \mathcal{L}}{\partial \phi_{f, a}}, \phi_{f, a}\right\rangle+\frac{\partial \mathcal{L}}{\partial f},\left\langle\frac{\partial \mathcal{L}}{\partial \phi_{f, a}}, \phi_{f, a}\right\rangle+\frac{\partial \mathcal{L}}{\partial a}\right) \\
& =\left\langle\frac{\partial \mathcal{L}}{\partial \phi_{f, a}}, \phi_{f, a}\right\rangle(1,1)+\left(\frac{\partial \mathcal{L}}{\partial f}, \frac{\partial \mathcal{L}}{\partial a}\right) .
\end{aligned}
$$

In this equality, for $\psi$ solution of (18)-(20) the first term in the right-hand side is zero, so that we obtain

$$
\begin{aligned}
\langle\nabla \tilde{J},(\delta f, \delta a)\rangle= & \langle\nabla \mathcal{L},(\delta f, \delta a)\rangle=-\langle\nabla E,(\delta f, \delta a)\rangle \\
= & \int_{Q_{T}}\left(\delta f\left(\phi_{f, a}\right) \frac{\partial \psi}{\partial z}, a\left(\phi_{f, a}\right) \frac{\partial^{2} \psi}{\partial z^{2}}\right) \\
& +\int_{z=L}\left(\delta f\left(\phi_{2}\right) \psi, a\left(\phi_{2}\right) \frac{\partial \psi}{\partial z}\right)-\int_{z=0}\left(0, a\left(\phi_{1}\right) \frac{\partial \psi}{\partial z}\right)(21)
\end{aligned}
$$

\section{Numerical schemes and discrete study}

We divide the interval $(0, L)$ into $M$ subintervals of length $\Delta z=L / M$ and the interval $(0, T)$ into $N$ subintervals of length $\Delta t=T / N$. For $n=0, \ldots, N$ and $j=0, \ldots, M$ we will denote by $\phi_{j}^{n}$ the value of numerical solution at $(j \Delta z, n \Delta t)$ and by $\phi_{j}^{0}, \phi_{2}^{n}, \phi_{j}^{\text {obs }}$ the corresponding approximation of $\phi_{0}, \phi_{2}, \phi^{o b s}$, respectively.

At discrete level the minimization corresponds to the following problem

$$
\min _{\mathbf{e}} J_{\Delta}\left(\phi_{j}^{n}(\mathbf{e})\right)
$$

where $J_{\Delta}$ is the discrete form of cost function. In the case of (12) is given by

$$
J_{\Delta}\left(\phi_{j}^{n}(\mathbf{e})\right)=\frac{1}{2} \sum_{j=0}^{M} \Delta z\left|\phi_{j}^{N}-\phi_{j}^{o b s}\right|^{2} .
$$


In this way we define the discrete Lagrangian associated by

$$
\mathcal{L} .\left(\phi_{j}^{n}, \psi_{j}^{n} ; \mathbf{e}\right)=\frac{1}{\Delta z} J_{\Delta}\left(\phi_{j}^{n}\right)-E_{\Delta}\left(\phi_{j}^{n}, \psi_{j}^{n} ; \mathbf{e}\right),
$$

where $E_{\Delta}\left(\phi_{j}^{n}, \psi_{j}^{n} ; \mathbf{e}\right)$ denotes a discrete weak formulation, it will be obtained by summation by parts of the numerical scheme by means we calculated the numerical solution of IBVP and $\psi_{j}^{n}$ will be chosen as solution of a discrete adjoint problem.

\subsection{First and second order explicit EO scheme}

In [9] the IBVP was well discretized by means of the first and second order EngquistOsher scheme. The first order EO scheme is

$$
\begin{aligned}
\frac{\phi_{j}^{n+1}-\phi_{j}^{n}}{\Delta t} & +q(n \Delta t) \frac{\phi_{j+1}^{n}-\phi_{j}^{n}}{\Delta z}+\frac{f_{b k}^{E O}\left(\phi_{j}^{n}, \phi_{j+1}^{n}\right)-f_{b k}^{E O}\left(\phi_{j-1}^{n}, \phi_{j}^{n}\right)}{\Delta z} \\
& =\frac{A\left(\phi_{j+1}^{n}\right)-2 A\left(\phi_{j}^{n}\right)+A\left(\phi_{j-1}^{n}\right)}{(\Delta z)^{2}}, j=1, \ldots, M-1,
\end{aligned}
$$

where

$$
\begin{aligned}
f_{b k}^{E O}\left(\phi_{j}^{n}, \phi_{j+1}^{n}\right) & =f_{b k}^{+}\left(\phi_{j}^{n}\right)+f_{b k}^{-}\left(\phi_{j+1}^{n}\right) \\
& =\left[f_{b k}(0)+\int_{0}^{\phi_{j}^{n}} \max \left(f_{b k}^{\prime}(s), 0\right) d s\right]+\left[\int_{0}^{\phi_{j+1}^{n}} \min \left(f_{b k}^{\prime}(s), 0\right) d s\right] .
\end{aligned}
$$

The second order EO scheme is defined by

$$
\begin{aligned}
\frac{\phi_{j}^{n+1}-\phi_{j}^{n}}{\Delta t} & +q(n \Delta t) \frac{\phi_{j+1}^{L}-\phi_{j}^{R}}{\Delta z}+\frac{f_{b k}^{E O}\left(\phi_{j}^{R}, \phi_{j+1}^{L}\right)-f_{b k}^{E O}\left(\phi_{j-1}^{R}, \phi_{j}^{L}\right)}{\Delta z} \\
& =\frac{A\left(\phi_{j+1}^{n}\right)-2 A\left(\phi_{j}^{n}\right)+A\left(\phi_{j-1}^{n}\right)}{(\Delta z)^{2}}, j=1, \ldots, M-1,
\end{aligned}
$$

where

$$
\phi_{j}^{L}=\phi_{j}^{n}-\frac{\Delta z}{2} s_{j}^{n} \quad \text { and } \quad \phi_{j}^{R}=\phi_{j}^{n}+\frac{\Delta z}{2} s_{j}^{n} .
$$

Here $s_{1}^{n}=s_{M-1}^{n}=0$ and for $j=2, \ldots, M-2$ we have

$$
s_{j}^{n}=\operatorname{mm}\left(\theta \frac{\phi_{j}^{n}-\phi_{j-1}^{n}}{\Delta z}, \frac{\phi_{j+1}^{n}-\phi_{j-1}^{n}}{2 \Delta z}, \theta \frac{\phi_{j+1}^{n}-\phi_{j}^{n}}{\Delta z}\right), \quad \theta \in[0,2],
$$

with $\mathrm{mm}$ the minmod function

$$
\operatorname{mm}(a, b, c)= \begin{cases}\min (a, b, c), & \text { if } a, b, c>0 \\ \max (a, b, c), & \text { if } a, b, c<0 \\ 0, & \text { otherwise }\end{cases}
$$

In both cases, first and second order, $\phi_{M}^{n}$ is obtained by $\phi_{M}^{n}=\phi_{2}(n \Delta t)$ and $\phi_{0}^{n}$ by the following formula

$$
\frac{\phi_{0}^{n+1}-\phi_{0}^{n}}{\Delta t}+q(n \Delta t) \frac{\phi_{1}^{n}-\phi_{0}^{n}}{\Delta z}+\frac{f_{b k}^{E O}\left(\phi_{0}^{n}, \phi_{1}^{n}\right)}{\Delta z}=\frac{A\left(\phi_{1}^{n}\right)-A\left(\phi_{0}^{n}\right)}{(\Delta z)^{2}}
$$


Denoting by $\lambda=\Delta t / \Delta z$ and $\nu=\Delta t /(\Delta z)^{2}$ the discrete weak formulation for (22) is given by

$$
\begin{aligned}
E_{\Delta}\left(\phi_{j}^{n}, \psi_{j}^{n}, \mathbf{e}\right)= & \sum_{n, j}\left\{\phi_{j}^{n+1}-\phi_{j}^{n}+\lambda q(n \Delta t)\left(\phi_{j+1}^{n}-\phi_{j}^{n}\right)+\lambda\left(f_{b k}^{E O}\left(\phi_{j}^{n}, \phi_{j+1}^{n}, \mathbf{e}\right)\right.\right. \\
& \left.\left.-f_{b k}^{E O}\left(\phi_{j-1}^{n}, \phi_{j}^{n}, \mathbf{e}\right)\right)-\nu\left(A\left(\phi_{j+1}^{n}, \mathbf{e}\right)-2 A\left(\phi_{j}^{n}, \mathbf{e}\right)+A\left(\phi_{j-1}^{n}, \mathbf{e}\right)\right)\right\} \psi_{j}^{n+1} \\
= & \sum_{n, j}\left\{\phi_{j}^{n}\left[\psi_{j}^{n}-\psi_{j}^{n+1}+\lambda q(n \Delta t)\left(\psi_{j-1}^{n+1}-\psi_{j}^{n+1}\right)\right]\right. \\
& \left.+\lambda f_{b k}^{E O}\left(\phi_{j}^{n}, \phi_{j+1}^{n}\right)\left(\psi_{j}^{n+1}-\psi_{j+1}^{n+1}\right)-\nu A\left(\phi_{j}^{n}\right)\left(\psi_{j-1}^{n+1}-2 \psi_{j}^{n+1}+\psi_{j+1}^{n+1}\right)\right\} \\
& +\sum_{j=0}^{M-1}\left\{\phi_{j}^{N} \psi_{j}^{N}-\phi_{j}^{0} \psi_{j}^{0}\right\} \\
& +\sum_{n=0}^{N-1}\left\{\lambda\left[q(n \Delta t) \phi_{M}^{n} \psi_{M-1}^{n+1}-q(n \Delta t) \phi_{0}^{n} \psi_{-1}^{n+1}+f_{b k}^{E O}\left(\phi_{M-1}^{n}, \phi_{M}^{n}\right) \psi_{M}^{n+1}\right]\right. \\
& \left.-\nu\left[A\left(\phi_{M}^{n}\right) \psi_{M-1}^{n+1}-A\left(\phi_{M-1}^{n}\right) \psi_{M}^{n+1}-A\left(\phi_{0}^{n}\right)\left(\psi_{-1}^{n+1}-\psi_{0}^{n+1}\right)\right]\right\},
\end{aligned}
$$

where we approximated the boundary condition at $z=0$ by

$$
\left.f_{b k}(\phi)\left(1+\frac{\sigma^{\prime}(\phi)}{\Delta \rho g \phi} \frac{\partial \phi}{\partial z}\right)\right|_{z=0} \approx f_{b k}^{E O}\left(\phi_{-1}^{n}, \phi_{0}^{n}\right)-\frac{A\left(\phi_{0}^{n}\right)-A\left(\phi_{-1}^{n}\right)}{\Delta z}=0 .
$$

Taking the derivative of $E_{\Delta}$ with respect to $\phi_{j}^{n}$ we obtain

$$
\begin{aligned}
\frac{\partial E_{\Delta}}{\partial \phi_{j}^{n}}=\psi_{j}^{n} & -\psi_{j}^{n+1}+\lambda q(n \Delta t)\left(\psi_{j-1}^{n+1}-\psi_{j}^{n+1}\right) \\
& +\lambda\left\{\min \left(f_{b k}^{\prime}\left(\phi_{j}^{n}\right), 0\right) \psi_{j-1}^{n+1}+\left|f_{b k}^{\prime}\left(\phi_{j}^{n}\right)\right| \psi_{j}^{n+1}-\max \left(f_{b k}^{\prime}\left(\phi_{j}^{n}\right), 0\right) \psi_{j+1}^{n+1}\right\} \\
& -\nu a\left(\phi_{j}^{n}\right)\left(\psi_{j-1}^{n+1}-2 \psi_{j}^{n+1}+\psi_{j+1}^{n+1}\right)+\psi_{j}^{N} \delta_{n, N} \\
& -\left[\lambda q(n \Delta t) \psi_{-1}^{n+1}-\nu a\left(\phi_{0}^{n}\right)\left(\psi_{-1}^{n+1}-\psi_{0}^{n+1}\right)\right] \delta_{j, 0} \\
& +\left[\lambda \max \left(f_{b k}^{\prime}\left(\phi_{M-1}^{n}\right), 0\right)+\nu a\left(\phi_{M-1}^{n}\right)\right] \psi_{M}^{n+1} \delta_{j, M-1} .
\end{aligned}
$$

If we consider that $\left(\partial \mathcal{L}_{\Delta}\right) /\left(\partial \phi_{j}^{n}\right)$ should be zero we have the follow adjoint scheme to $(22)$

$$
\begin{aligned}
\frac{\psi_{j}^{n}-\psi_{j}^{n+1}}{\Delta t}+q(n \Delta t) \frac{\psi_{j-1}^{n+1}-\psi_{j}^{n+1}}{\Delta z}+\frac{F A_{1}}{\Delta z} & =\frac{a\left(\phi_{j}^{n}\right)\left(\psi_{j-1}^{n+1}-2 \psi_{j}^{n+1}+\psi_{j+1}^{n+1}\right)}{(\Delta z)^{2}}, \\
\psi_{j}^{N} & =\frac{\partial J_{\Delta}}{\partial \phi_{j}^{N}}, \\
q(n \Delta t) \psi_{-1}^{n+1}-a\left(\phi_{0}^{n}\right) \frac{\psi_{-1}^{n+1}-\psi_{0}^{n+1}}{\Delta z} & =0 \\
{\left[\lambda \max \left(f_{b k}^{\prime}\left(\phi_{M-1}^{n}\right), 0\right)+\nu a\left(\phi_{M-1}^{n}\right)\right] \psi_{M}^{n+1} } & =0
\end{aligned}
$$

where

$$
F A_{1}=\min \left(f_{b k}^{\prime}\left(\phi_{j}^{n}\right), 0\right) \psi_{j-1}^{n+1}+\left|f_{b k}^{\prime}\left(\phi_{j}^{n}\right)\right| \psi_{j}^{n+1}-\max \left(f_{b k}^{\prime}\left(\phi_{j}^{n}\right), 0\right) \psi_{j+1}^{n+1} .
$$


Thus, we obtain the discrete gradient

$$
\begin{aligned}
\nabla \hat{J}_{\Delta}(\mathbf{e})= & -\nabla_{\mathbf{e}} E_{\Delta} \Delta z \\
= & -\sum_{n, j} \Delta t \nabla_{\mathbf{e}} f_{b k}^{E O}\left(\phi_{j}^{n}, \phi_{j+1}^{n}\right)\left(\psi_{j}^{n+1}-\psi_{j+1}^{n+1}\right) \\
& -\lambda \nabla_{\mathbf{e}} A\left(\phi_{j}^{n}\right)\left(\psi_{j-1}^{n+1}-2 \psi_{j}^{n+1}+\psi_{j+1}^{n+1}\right)-\sum_{n=0}^{N-1}\left\{\Delta t \nabla_{\mathbf{e}} f_{b k}^{E O}\left(\phi_{M-1}^{n}, \phi_{M}^{n}\right) \psi_{M}^{n+1}\right. \\
& \left.-\lambda\left[\nabla_{\mathbf{e}} A\left(\phi_{M}^{n}\right) \psi_{M-1}^{n+1}-\nabla_{\mathbf{e}} A\left(\phi_{M-1}^{n}\right) \psi_{M}^{n+1}-\nabla_{\mathbf{e}} A\left(\phi_{0}^{n}\right)\left(\psi_{-1}^{n+1}-\psi_{0}^{n+1}\right)\right]\right\} .
\end{aligned}
$$

If we use (23) instead of (22) we get the adjoint scheme

$$
\begin{aligned}
\frac{\psi_{j}^{n}-\psi_{j}^{n+1}}{\Delta t}+\frac{q(n \Delta t) F q}{\Delta z}+\frac{F A_{2}}{\Delta z} & =\frac{a\left(\phi_{j}^{n}\right)\left(\psi_{j-1}^{n+1}-2 \psi_{j}^{n+1}+\psi_{j+1}^{n+1}\right)}{\Delta z^{2}} \\
\psi_{j}^{N} & =\frac{\partial J_{\Delta}}{\partial \phi_{j}^{N}} \\
q\left(n_{\Delta} t\right) \frac{\partial \phi_{0}^{L}}{\partial \phi_{0}^{n}} \psi_{-1}^{n+1}-a\left(\phi_{0}^{n}\right) \frac{\psi_{-1}^{n+1}-\psi_{0}^{n+1}}{\Delta z} & =0
\end{aligned}
$$

$\lambda \max \left(f_{b k}^{\prime}\left(\phi_{M-1}^{R}\right), 0\right) \frac{\partial \phi_{M-1}^{R}}{\partial \phi_{0}^{n}} \psi_{M-1}^{n+1}+\nu a\left(\phi_{M-1}^{n}\right) \psi_{M}^{n+1}=0$,

where

$$
\begin{aligned}
F A_{2}= & \min \left(f_{b k}^{\prime}\left(\phi_{j-1}^{L}\right), 0\right) \frac{\partial \phi_{j-1}^{L}}{\partial \phi_{j}^{n}}\left(\psi_{j-2}^{n+1}-\psi_{j-1}^{n+1}\right) \\
& +\left\{\max \left(f_{b k}^{\prime}\left(\phi_{j-1}^{R}\right), 0\right) \frac{\partial \phi_{j-1}^{R}}{\partial \phi_{j}^{n}}+\min \left(f_{b k}^{\prime}\left(\phi_{j}^{L}\right), 0\right) \frac{\partial \phi_{j}^{L}}{\partial \phi_{j}^{n}}\right\}\left(\psi_{j-1}^{n+1}-\psi_{j}^{n+1}\right) \\
& +\left\{\max \left(f_{b k}^{\prime}\left(\phi_{j}^{R}\right), 0\right) \frac{\partial \phi_{j}^{R}}{\partial \phi_{j}^{n}}+\min \left(f_{b k}^{\prime}\left(\phi_{j+1}^{L}\right), 0\right) \frac{\partial \phi_{j+1}^{L}}{\partial \phi_{j}^{n}}\right\}\left(\psi_{j}^{n+1}-\psi_{j+1}^{n+1}\right) \\
& +\max \left(f_{b k}^{\prime}\left(\phi_{j+1}^{R}\right), 0\right) \frac{\partial \phi_{j+1}^{R}}{\partial \phi_{j}^{n}}\left(\psi_{j+1}^{n+1}-\psi_{j+2}^{n+1}\right), \\
F q= & \left(\frac{\partial \phi_{j-1}^{L}}{\partial \phi_{j}^{n}}-\frac{\partial \phi_{j-1}^{R}}{\partial \phi_{j}^{n}}\right)\left(\psi_{j-2}^{n+1}-\psi_{j-1}^{n+1}\right)+\left(\frac{\partial \phi_{j}^{L}}{\partial \phi_{j}^{n}}-\frac{\partial \phi_{j}^{R}}{\partial \phi_{j}^{n}}\right)\left(\psi_{j-1}^{n+1}-\psi_{j}^{n+1}\right) \\
+ & \left(\frac{\partial \phi_{j+1}^{L}}{\partial \phi_{j}^{n}}-\frac{\partial \phi_{j+1}^{R}}{\partial \phi_{j}^{n}}\right)\left(\psi_{j}^{n+1}-\psi_{j+1}^{n+1}\right) .
\end{aligned}
$$

In this case the gradient is given by

$$
\begin{aligned}
\nabla \hat{J}_{\Delta}(\mathbf{e})= & -\sum_{n, j}\left\{\Delta t \nabla \mathbf{e} f_{b k}^{E 0}\left(\phi_{j}^{R}, \phi_{j+1}^{L}\right)\left(\psi_{j}^{n+1}-\psi_{j+1}^{n+1}\right)\right. \\
& \left.-\lambda \nabla \mathbf{e} A\left(\phi_{j}^{n}\right)\left(\psi_{j+1}^{n+1}-2 \psi_{j}^{n+1}+\psi_{j+1}^{n+1}\right)\right\}-\sum_{n=0}^{N-1}\left\{\Delta t \nabla \mathbf{e} f_{b k}^{E 0}\left(\phi_{M-1}^{R}, \phi_{M}^{L}\right) \psi_{M-1}^{n+1}\right. \\
& \left.-\lambda\left[\nabla \mathbf{e} A\left(\phi_{M}^{n}\right) \psi_{M-1}^{n+1}-\nabla \mathbf{e} A\left(\phi_{M-1}^{n}\right) \psi_{M}^{n+1}-\nabla \mathbf{e} A\left(\phi_{0}^{n}\right)\left(\psi_{0}^{n+1}-\psi_{-1}^{n+1}\right)\right]\right\} .
\end{aligned}
$$


If the following CFL condition

$$
\lambda \max _{t \in[0, T]}|q(t)|+\lambda \max _{\phi \in\left[0, \phi_{\max }\right]}\left|f_{b k}^{\prime}(\phi)\right|+2 \nu \max _{\phi \in\left[\phi_{c}, \phi_{\max }\right]}|a(\phi)| \leq 1
$$

is satisfied, then the first and second order schemes are stable. For another CFL condition see [9].

\subsection{Implicit and semi-implicit schemes}

The direct problem can be discretized by semi-implicitly scheme

$$
\begin{aligned}
\frac{\phi_{j}^{n+1}-\phi_{j}^{n}}{\Delta t} & +q(n \Delta t) \frac{\phi_{j+1}^{n}-\phi_{j}^{n}}{\Delta z}+\frac{f_{b k}^{E O}\left(\phi_{j}^{n}, \phi_{j+1}^{n}\right)-f_{b k}^{E O}\left(\phi_{j-1}^{n}, \phi_{j}^{n}\right)}{\Delta z} \\
& =\frac{A\left(\phi_{j+1}^{n+1}\right)-2 A\left(\phi_{j}^{n+1}\right)+A\left(\phi_{j-1}^{n+1}\right)}{(\Delta z)^{2}}, \quad j=1, \ldots, M-1
\end{aligned}
$$

or by the implicit scheme

$$
\begin{aligned}
\frac{\phi_{j}^{n+1}-\phi_{j}^{n}}{\Delta t} & +q((n+1) \Delta t) \frac{\phi_{j+1}^{n+1}-\phi_{j}^{n+1}}{\Delta z}+\frac{f_{b k}^{E O}\left(\phi_{j}^{n+1}, \phi_{j+1}^{n+1}\right)-f_{b k}^{E O}\left(\phi_{j-1}^{n+1}, \phi_{j}^{n+1}\right)}{\Delta z} \\
& =\frac{A\left(\phi_{j+1}^{n+1}\right)-2 A\left(\phi_{j}^{n+1}\right)+A\left(\phi_{j-1}^{n+1}\right)}{(\Delta z)^{2}}, \quad j=1, \ldots, M-1
\end{aligned}
$$

In both cases the boundary condition at $z=L$ is discretized by $\phi_{M}^{n+1}=\phi_{2}((n+1) \Delta t)$ while the boundary condition at $z=0$ is calculated by the interior scheme with the following approximation

$$
\left.\left(f_{b k}(u)-\frac{\partial A(u)}{\partial z}\right)\right|_{z=0} \approx f_{b k}^{E O}\left(\phi_{-1}^{n+1}, \phi_{0}^{n+1}\right)-\frac{A\left(\phi_{0}^{n+1}\right)-A\left(\phi_{-1}^{n+1}\right)}{\Delta z}=0 .
$$

Applying the same strategy as the previous section for to calculate the gradient, we have the adjoint state

$$
\begin{aligned}
\frac{\psi_{j}^{n}-\psi_{j}^{n+1}}{\Delta t}+q(n \Delta t) \frac{\psi_{j-1}^{n}-\psi_{j}^{n}}{\Delta t}+\frac{F A_{1}}{\Delta z} & =a\left(\phi_{j}^{n}\right) \frac{\psi_{j-1}^{n}-2 \psi_{j}^{n}+\psi_{j+1}^{n}}{(\Delta z)^{2}} \\
\left(1+2 \nu a\left(\phi_{j}^{N}\right)\right) \psi_{j}^{N} & =\frac{\partial J_{\Delta}}{\partial \phi_{j}^{N}} \\
q(n \Delta t) \psi_{-1}^{n+1}-a\left(\phi_{0}^{n}\right) \frac{\psi_{-1}^{n}-\psi_{0}^{n}}{\Delta z} & =0 \\
\lambda \max \left\{f_{b k}^{\prime}\left(\phi_{M-1}^{n}\right), 0\right\} \psi_{M}^{n+1}+\nu a\left(\phi_{M-1}^{n}\right) \psi_{M}^{n} & =0
\end{aligned}
$$

for (25) and

$$
\begin{aligned}
\frac{\psi_{j}^{n}-\psi_{j}^{n+1}}{\Delta t}+q(n \Delta t) \frac{\psi_{j-1}^{n}-\psi_{j}^{n}}{\Delta t}+\frac{F A I}{\Delta z} & =a\left(\phi_{j}^{n}\right) \frac{\psi_{j-1}^{n}-2 \psi_{j}^{n}+\psi_{j+1}^{n}}{(\Delta z)^{2}} \\
\psi_{j}^{N} & =\frac{\partial J_{\Delta}}{\partial \phi_{j}^{N-1}} \\
q(n \Delta t) \psi_{-1}^{n}-a\left(\phi_{0}^{n}\right) \frac{\psi_{-1}^{n}-\psi_{0}^{n}}{\Delta z} & =0 \\
\left\{\lambda \max \left\{f_{b k}^{\prime}\left(\phi_{M-1}^{n}\right), 0\right\}+\nu a\left(\phi_{M-1}^{n}\right)\right\} \psi_{M}^{n} & =0
\end{aligned}
$$


for (26), where $F A_{1}$ is given by (24) and

$$
F A I=\min \left(f_{b k}^{\prime}\left(\phi_{j}^{n}\right), 0\right) \psi_{j-1}^{n}+\left|f_{b k}^{\prime}\left(\phi_{j}^{n}\right)\right| \psi_{j}^{n}-\max \left(f_{b k}^{\prime}\left(\phi_{j}^{n}\right), 0\right) \psi_{j+1}^{n} .
$$

The gradient in the case of semi-implicit is given by

$$
\begin{aligned}
\nabla \hat{J}_{\Delta}(\mathbf{e})= & -\nabla_{\mathbf{e}} E_{\Delta} \Delta z \\
= & -\sum_{n, j} \Delta t \nabla \mathbf{e} f_{b k}^{E O}\left(\phi_{j}^{n}, \phi_{j+1}^{n}\right)\left(\psi_{j}^{n+1}-\psi_{j+1}^{n+1}\right)-\lambda \nabla \mathbf{e} A\left(\phi_{j}^{n}\right)\left(\psi_{j-1}^{n}-2 \psi_{j}^{n}+\psi_{j+1}^{n}\right) \\
& -\sum_{n=0}^{N-1}\left\{\Delta t \nabla \mathbf{e} f_{b k}^{E O}\left(\phi_{M-1}^{n}, \phi_{M}^{n}\right) \psi_{M}^{n+1}\right. \\
& \left.-\lambda\left[\nabla_{\mathbf{e}} A\left(\phi_{M}^{n}\right) \psi_{M-1}^{n}-\nabla_{\mathbf{e}} A\left(\phi_{M-1}^{n}\right) \psi_{M}^{n}-\nabla_{\mathbf{e}} A\left(\phi_{0}^{n}\right)\left(\psi_{-1}^{n}-\psi_{0}^{n}\right)\right]\right\},
\end{aligned}
$$

whereas in the implicit ones is

$$
\begin{aligned}
\nabla \hat{J}_{\Delta}(\mathbf{e})= & -\nabla_{\mathbf{e}} E_{\Delta} \Delta z \\
= & -\sum_{n, j} \Delta t \nabla_{\mathbf{e}} f_{b k}^{E O}\left(\phi_{j}^{n}, \phi_{j+1}^{n}\right)\left(\psi_{j}^{n}-\psi_{j+1}^{n}\right)-\lambda \nabla \mathbf{e} A\left(\phi_{j}^{n}\right)\left(\psi_{j-1}^{n}-2 \psi_{j}^{n}+\psi_{j+1}^{n}\right) \\
& -\sum_{n=0}^{N-1}\left\{\Delta t \nabla_{\mathbf{e}} f_{b k}^{E O}\left(\phi_{M-1}^{n}, \phi_{M}^{n}\right) \psi_{M}^{n}\right. \\
& \left.-\lambda\left[\nabla \mathbf{e} A\left(\phi_{M}^{n}\right) \psi_{M-1}^{n}-\nabla \mathbf{e} A\left(\phi_{M-1}^{n}\right) \psi_{M}^{n}-\nabla_{\mathbf{e}} A\left(\phi_{0}^{n}\right)\left(\psi_{-1}^{n}-\psi_{0}^{n}\right)\right]\right\}
\end{aligned}
$$

The CFL condition for the semi-implicit scheme is given by

$$
\lambda\left(\max _{t \in[0, T]}|q(t)|+\max _{\phi \in\left[0, \phi_{\max }\right]}\left|f_{b k}^{\prime}(\phi)\right|\right) \leq 1
$$

Meanwhile, the implicit scheme is "CFL free", see [9].

The unconditional stability of the implicit scheme is useful for the reason that it allows the choose of a coarse grid without loosing the convergence to the entropy solution. Therefore, the implicit scheme is a good alternative to simulate numerically the physical problem with a few (reasonable) steps of time to overcome the cumbersome number of steps of time in the explicit and semi-implicit schemes.

\subsection{Numerical tests}

5.3.1. Identification from analytic data. Let us consider $f_{b k}$ and $\sigma_{e}$ like (13) and (14) with the value of the parameters given by the Table 1 . Then the function $\phi_{1}(z, t)=z^{2}+(t / 30000)^{2}$ is solution of the following IBVP

$$
\begin{aligned}
& \frac{\partial \phi}{\partial t}+\frac{\partial}{\partial z}\left(f_{b k}(\phi)\right)=\frac{\partial^{2} A(\phi)}{\partial^{2} z}+g_{1}(z, t) \quad(z, t) \in Q_{T} \\
& \phi(z, 0) \quad=0.05 \quad z \in[0,1] \\
& \phi(1, t) \quad=\phi_{1}(1, t) \quad t \in[0, T] \\
& f_{b k}(\phi)-\left.\frac{\partial A(\phi)}{\partial z}\right|_{z=0}=b_{1}(t) \quad t \in[0, T]
\end{aligned}
$$


Table 1. Parameters for direct simulation [20].

\begin{tabular}{|c|c|c|c|}
\hline$C$ & $\alpha$ & $\beta$ & $\phi_{c}$ \\
\hline 15.6 & 5.0 & 6.0 & 0.1 \\
\hline
\end{tabular}

Table 2. Identified parameters with the second order EO explicit scheme.

\begin{tabular}{|c|c|c|c|c|c|}
\hline$\phi^{\text {obs }}$ & $\mathrm{J}$ & $C$ & $\alpha$ & $\beta$ & $\phi_{c}$ \\
\hline$\phi_{1}$ & $8.245 \mathrm{e}-6$ & 16.09203 & 5.50039 & 6.50071 & 0.10053 \\
\hline $1.01 \phi_{1}$ & $4.553 \mathrm{e}-6$ & 16.08856 & 5.50039 & 6.50069 & 0.10280 \\
\hline
\end{tabular}

where the source term $g_{1}$ is defined by

$$
g_{1}(z, t)=\frac{\partial \phi_{1}}{\partial t}+\frac{\partial}{\partial z}\left(f_{b k}\left(\phi_{1}\right)\right)-\frac{\partial^{2} A\left(\phi_{1}\right)}{\partial^{2} z}
$$

and the boundary condition $b_{1}$ is given by

$$
b_{1}(t)=f_{b k}\left(\phi_{1}(0, t)\right)-\frac{\partial A\left(\phi_{1}(0, t)\right)}{\partial z} .
$$

The identified parameters given at Table 2 are obtained with the second order explicit EO scheme $(\theta=1.0)$ and the observed data $\phi_{1}(z, 12000)$ and the noised form $1.01 \phi_{1}(z, 12000)$. The grid parameters are $M=200$ and $C F L=0.98$. The initial guess corresponds to $C=16.1, \alpha=5.5, \beta=6.5$ and $\phi_{c}=0.2$. See Figures 1 and 2 .

5.3.2. Identification from simulated data. We present here a validation of the above Lagrangian method as well as a comparison between the four numerical schemes developed. Since we did not have access to real experimental data, the idea consists in using as an observation the result of a direct simulation. We used the standard simulation given by Concha [20], which is very close to experimental data results. All tests are developed for batch sedimentation velocity $q=0$, with an initially homogeneous suspension of concentration, namely $\phi_{0}=0.05$. The column is assumed to be closed, that is $\phi_{2}=0$. The physical constants considered are $u_{\infty}=-1.7200 \times 10^{-4}, \phi_{\max }=0.7$, $\Delta \rho=1500$ and $g=9.81$. We summarize in Table 1 the parameters used for this simulation, which we want to recover in the inverse problem.

Three tests are performed, the first one is concerned only with the flux identification, that is parameter $C$. The second test identifies the diffusion together with the flux, that is parameters $C, \alpha$ and $\beta$, the critical concentration $\phi_{c}$ being fixed. Finally, we perform the complete identification on the four parameters. We start with the following values: $\mathbf{e}=(16.5,5.0,6.0,0.1), \mathbf{e}=(16.5,5.5,6.5,0.1)$ and $\mathbf{e}=(16.5,5.5,6.5,0.2)$ for the test 1 , test 2 and test 3 respectively. Other several initial points were considered with very similar and close results. The identification problem is solved at $T=12144$ seconds in the three cases, and a simulation at $T=30000$ seconds with the identified parameters is proposed. 


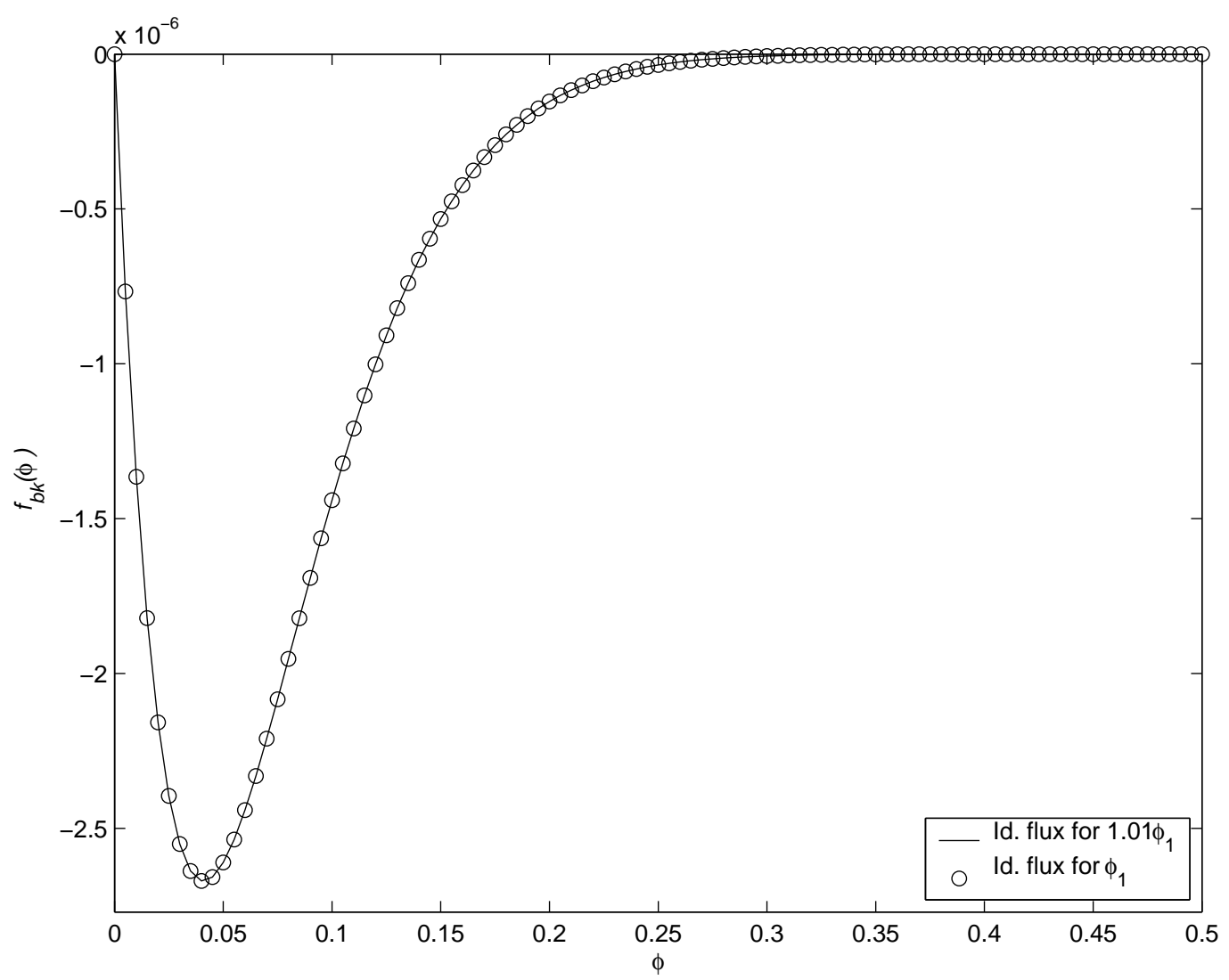

Figure 1. The identified flux for the observations $\phi_{1}$ and $1.01 \phi_{1}$, with the second order EO scheme at $T=12000$ with $\Delta x=0.005, C F L=0.98$ and $\theta=1$.

For each test, we compare the four numerical schemes presented above, namely the first order scheme EO1, the second order scheme EO2, the semi-implicit scheme EOS and the fully implicit scheme EOI. For the explicit and semi-implicit schemes we employ the $C F L=0.5$ and in the case of explicit second order scheme we consider $\theta=1$. For the implicit scheme we take $\Delta t=\Delta x$. Four different meshes were used, with $M=10,50,100,200$ steps.

In Figures 3-4 and table 3 we show numerical results for the first test. The Figures 5-6 and table 4 shows the results of the second test and the results for the third test are given in Figures 7-8 and table 5 .

All schemes give satisfactory results, and it should be emphasized that the value of the critical concentration $\phi_{c}$ is correctly recovered. The explicit scheme is of course the simplest to implement, but turns out to be worst one in terms of computational time. Indeed the stability restriction requires such a high number of time steps that the benefit of the computational simplicity is lost (here $\Delta t \approx \Delta x^{2}$ ). In the semi-explicit and fully implicit schemes, Gauss-Seidel and Newton methods are needed to solve linear and nonlinear systems, but this is compensated by the less restrictive CFL condition. For the semi-explicit the restriction becomes the same as in the hyperbolic case, that is $\Delta t \approx \Delta x$, and finally the implicit scheme is CFL free. 


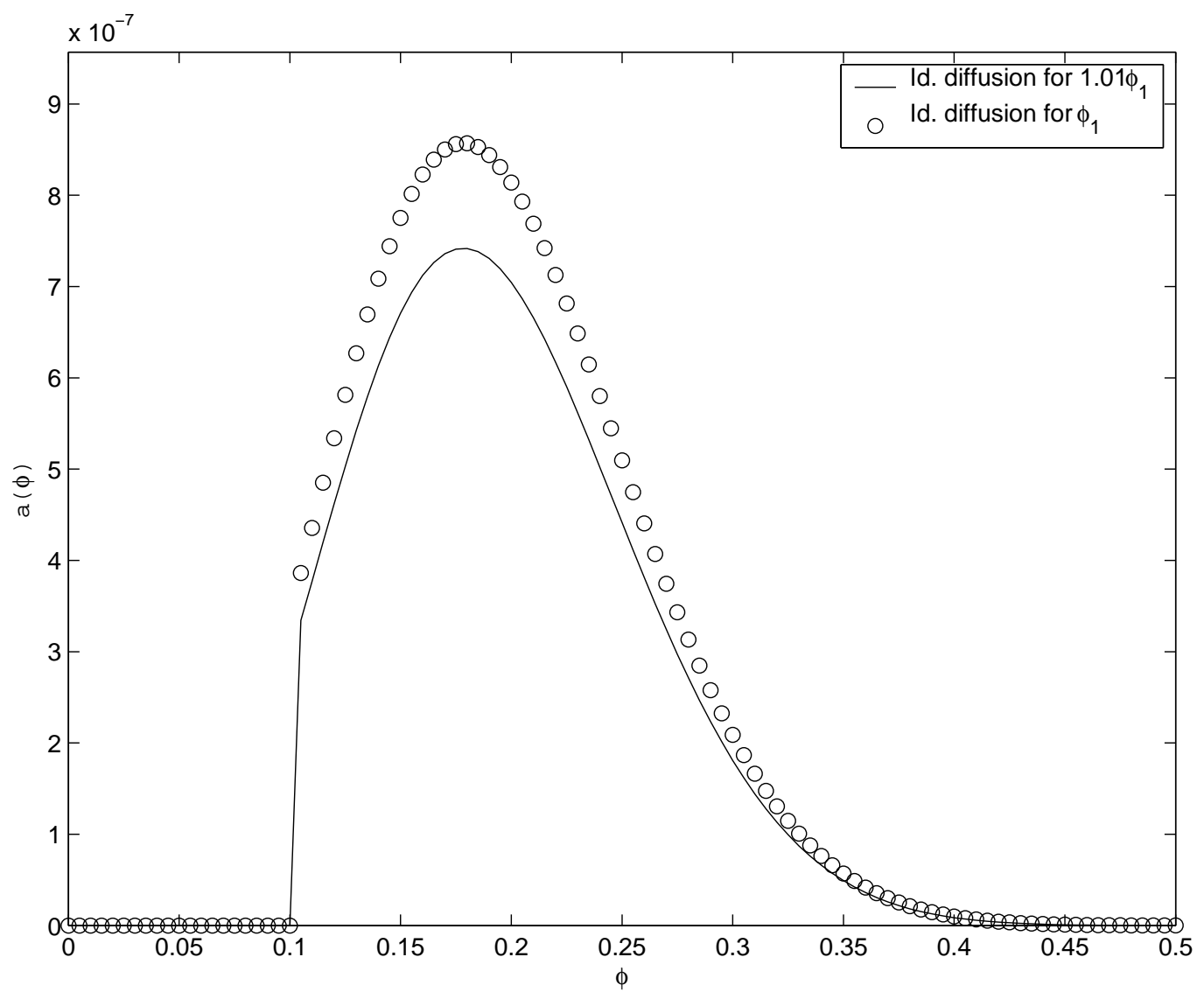

Figure 2. The identified diffusion for observations $\phi_{1}$ and $1.01 \phi_{1}$, with the second order EO scheme at $T=12000$ with $\Delta x=0.005, C F L=0.98$ and $\theta=1$.

Table 3. Identification of the flux: values of the parameter $C$.

\begin{tabular}{lllll}
\hline $\mathrm{M}$ & $\mathrm{EO} 1$ & $\mathrm{EO} 2$ & $\mathrm{EOS}$ & $\mathrm{EOI}$ \\
\hline 10 & 15.34225 & 15.78555 & 17.21670 & 14.00079 \\
50 & 15.62241 & 15.95830 & 15.50107 & 15.25519 \\
100 & 15.60076 & 15.77236 & 15.70925 & 15.39562 \\
200 & 15.58900 & 15.66536 & 15.64600 & 15.47951 \\
\hline
\end{tabular}

\section{Acknowledgments}

This work was supported by MECESUP UCO9907, FONDECYT 1000332, 7000332, 1030718, and Fondap in Applied Mathematics.

\section{References}

[1] Becker R., 1982 Espesamiento contínuo, diseño y simulación de espesadores, Engineering Thesis, Universidad de Concepción.

[2] Bürger R., Bustos M.C. and Concha F. 1999 Settling velocities of particulate systems: 9. 
Table 4. Identification of the flux and diffusion except the critical concentration $\phi_{c}$.

\begin{tabular}{lllll}
\hline $\mathrm{M}$ & Scheme & $\mathrm{C}$ & $\alpha$ & $\beta$ \\
\hline 10 & $\mathrm{EO} 1$ & 15.28574 & 5.48153 & 6.46587 \\
& $\mathrm{EO} 2$ & 15.73031 & 5.48855 & 6.47817 \\
& $\mathrm{EOS}$ & 17.02054 & 5.53600 & 6.38967 \\
& $\mathrm{EOI}$ & 13.92483 & 5.47128 & 6.46003 \\
\hline 50 & $\mathrm{EO1}$ & 15.59482 & 5.37159 & 6.07586 \\
& $\mathrm{EO} 2$ & 15.90027 & 5.43575 & 6.25493 \\
& EOS & 15.72207 & 5.47796 & 6.46396 \\
& EOI & 15.16180 & 5.45272 & 6.43251 \\
\hline 100 & EO1 & 15.54214 & 5.30271 & 6.12221 \\
& EO2 & 15.69580 & 5.42393 & 6.34055 \\
& EOS & 15.57037 & 5.44297 & 6.40494 \\
& EOI & 15.31271 & 5.37628 & 6.30522 \\
\hline 200 & EO1 & 15.59575 & 5.13254 & 5.86232 \\
& EO2 & 15.66016 & 5.30389 & 6.02822 \\
& EOS & 15.58839 & 5.27715 & 6.13142 \\
& EOI & 15.48579 & 5.12949 & 5.88291 \\
\hline
\end{tabular}

Table 5. Identification of the flux and diffusion.

\begin{tabular}{llllll}
\hline $\mathrm{M}$ & Scheme & $\mathrm{C}$ & $\alpha$ & $\beta$ & $\phi_{c}$ \\
\hline 10 & EO1 & 15.55524 & 5.50000 & 6.50000 & 0.20000 \\
& EO2 & 15.97224 & 5.50000 & 6.50000 & 0.20000 \\
& EOS & 17.67741 & 5.49970 & 6.49966 & 0.32618 \\
& EOI & 14.29822 & 5.50000 & 6.50000 & 0.20000 \\
\hline 50 & EO1 & 15.82567 & 5.50008 & 6.50008 & 0.10650 \\
& EO2 & 16.04526 & 5.50008 & 6.50008 & 0.10196 \\
& EOS & 16.05873 & 5.50019 & 6.50023 & 0.10593 \\
& EOI & 15.83821 & 5.50004 & 6.50003 & 0.11159 \\
\hline 100 & EO1 & 16.06191 & 5.50020 & 6.50023 & 0.10849 \\
& EO2 & 16.07651 & 5.50023 & 6.50026 & 0.10225 \\
& EOS & 16.08611 & 5.50028 & 6.50039 & 0.10886 \\
& EOI & 16.09204 & 5.50019 & 6.50021 & 0.11058 \\
\hline 200 & EO1 & 16.08896 & 5.50026 & 6.50035 & 0.10551 \\
& EO2 & 16.10939 & 5.50028 & 6.50037 & 0.10183 \\
& EOS & 16.09543 & 5.50031 & 6.50047 & 0.10635 \\
& EOI & 16.09969 & 5.50026 & 6.50034 & 0.10711 \\
\hline
\end{tabular}



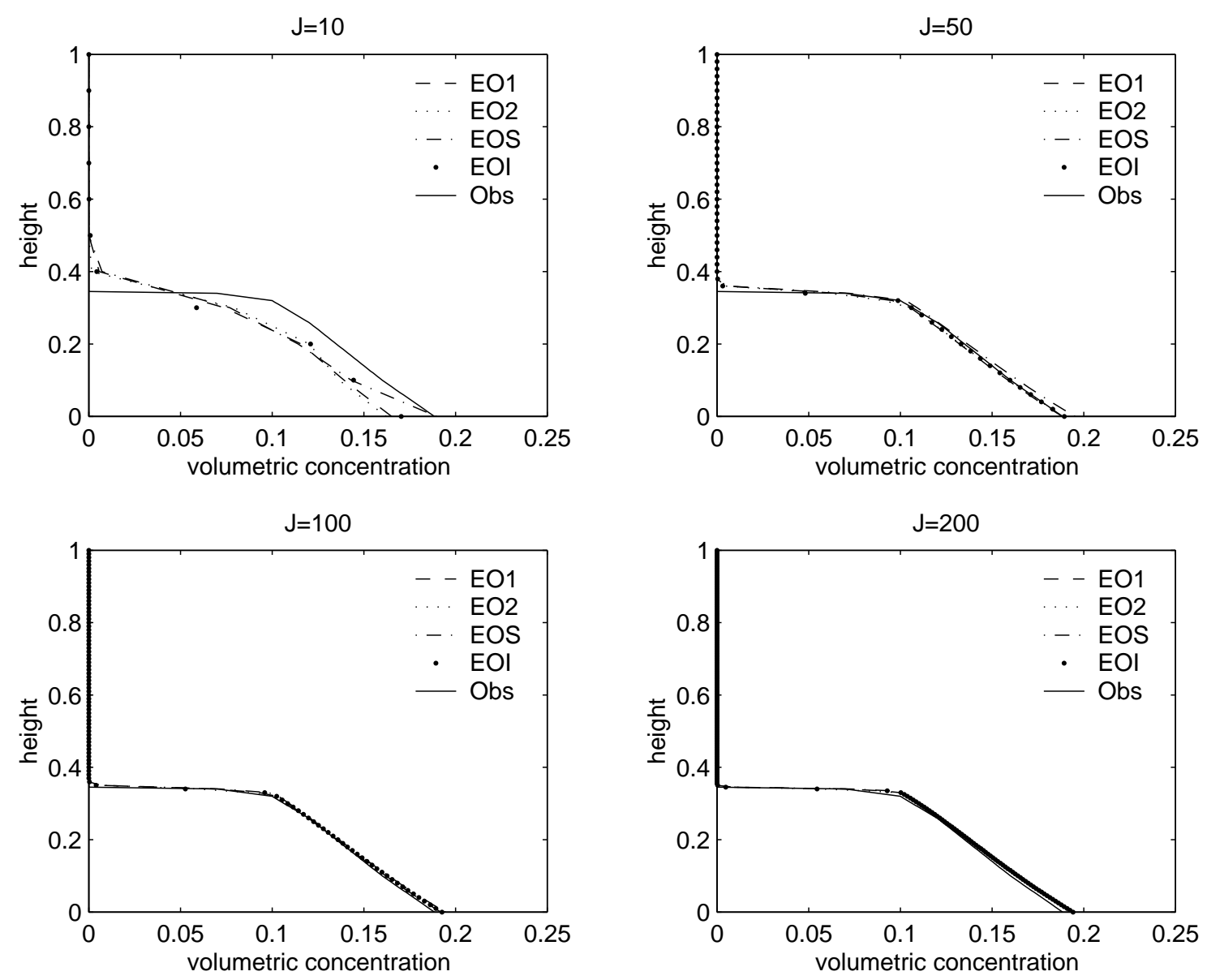

Figure 3. Numerical and observed concentration curves at $T=12144$ for test 1 .

Phenomenological theory of sedimentation processes: numerical simulation of flocculated suspensions in an ideal batch or continuous thickener Int. J. Miner. Process. 55 267-282.

[3] Bürger R. and Wendland W. L. 1998 Entropy boundary and jump conditions in the theory of Sedimentation with compression. Math. Meth. Appl. Sci. 21 865-882

[4] Bürger R. and Wendland W. L. 1998 Existence, uniqueness and stability of generalized solutions of an initial-boundary problem for a degenerating quasilinear parabolic equation. J. Math. Anal. Appl. 218 207-239

[5] Bürger R. and Concha F. 1998 Mathematical model and numerical simulation of the settling of flocculated suspensions Int. J. Multiphase Flow24 1005-1023

[6] Bürger R., Wendland W. L. and Concha F. 2000 Model equations for gravitational sedimentationconsolidation processes. ZAMM 80 79-92

[7] Bürger R., Evje S., Karlsen K. H. and Lie K.-A. 2000 Numerical methods for the simulation of the settling of flocculated suspensions. Chem. Eng. J. 80 91-104.

[8] Bürger R., Concha F., Fjelde K.-K. and Karlsen K. H. 2000 Numerical simulation of the settling of polydisperse suspensions of spheres. Powder Technol. 113 30-54.

[9] Bürger R. and Karlsen K. H. 2001 On some upwind difference schemes for the phenomenological sedimentation-consolidation process. J. Eng. Math. 41 145-166.

[10] Bürger R., Evje S. and Karlsen K. H.2000 On strongly convection-diffusion problems modeling sedimentation-consolidation processes J. Math. Anal. Appl. 247 517-556

[11] Bürger R., Concha F. and Tiller F. M. 2000 Applications of the phenomenological theory to several published experimental cases of sedimentation process. Chem. Eng. J. 80 105-117. 


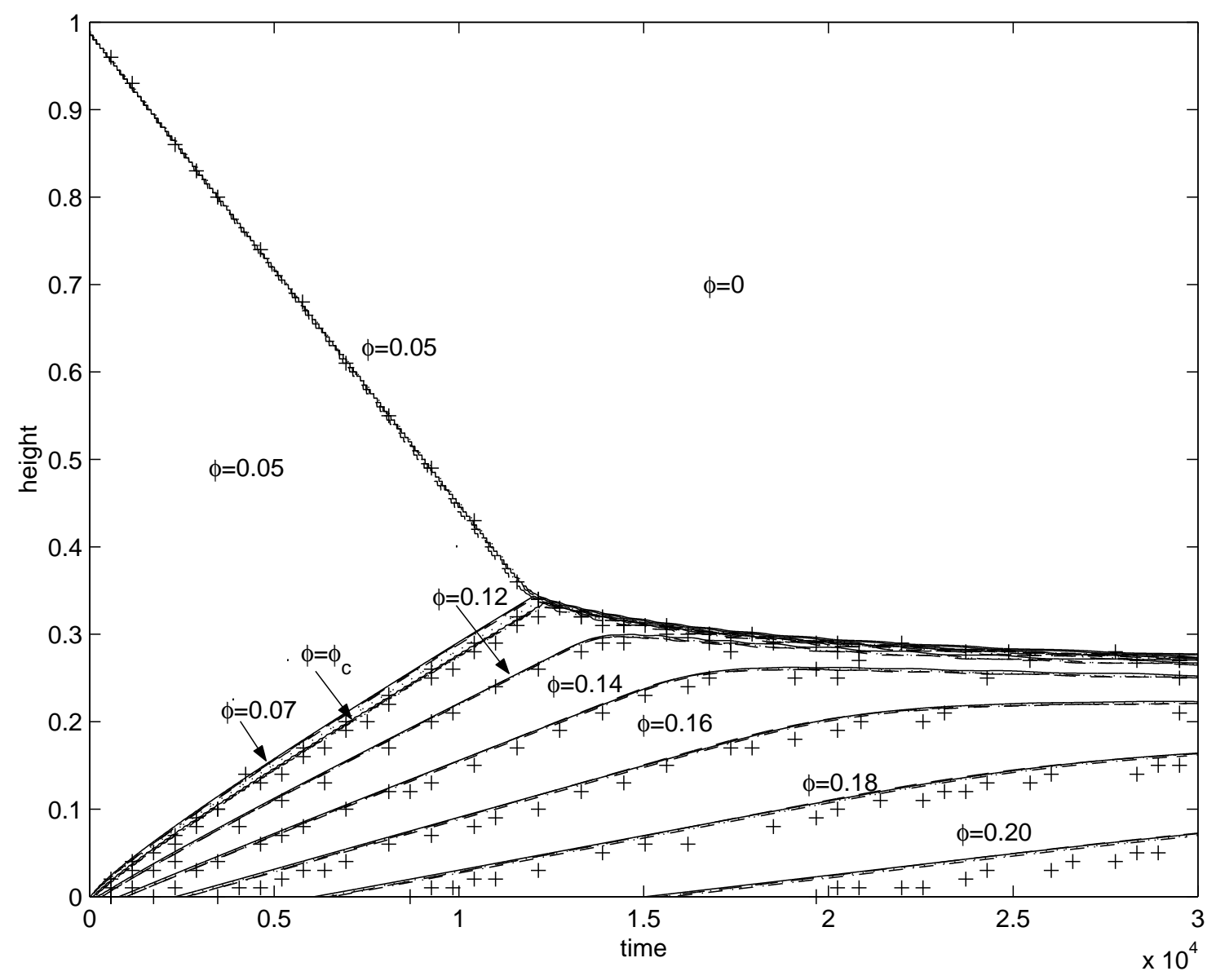

Figure 4. Isoconcentrations curves for test 1 with $M=200, T=30000$. We denote by . - .- the results for EO1, by $\cdots$ for EO2, by - for EOS, by - - for EOI and by +++ for the observation data.

[12] Bustos M. C. and F. Concha 1988 Simulation of batch sedimentation with compression. AIChE J. 34 859-861

[13] Bustos M. C., Concha F., Bürger R. and Tory M. 1999 Sedimentation and Thickening. (Dodrecht: Kluwer Academic Publishers)

[14] Cannon J. R. and Zachmann D. 1982 Parameter determination in parabolic partial differential equations from overspecified boundary data Int. J. Engng. Sci. 20 779-788

[15] Cannon J. R. 1964 Determination of certain parameters in heat conduction problems J. of Math. Anal. and Appl. 8 188-201

[16] Carrillo J. 1999 Entropy solutions for nonlinear degenerate problems Arch. Rational Mech. Anal. 147(4) 269-361

[17] Chavent G. 1979 Identification of distributed parameters: about the output least square method, its implementation, and identifiability Proceeding of the 5th. IFAC Symposium of Identification and System Parameter Estimations Pergamon Press, Vol 1 85-97

[18] Chavent G. and Cohen G. 1977 Numerical Approximations and Identification in 1-D Parabolic Degenerate non linear Diffusion and Transport Equation. Proceeding of the 7th. IFIP Conference on Optimization Methods, Würzburg, FRG

[19] Cockburn B. and Gripenberg G. 1999 Continuous dependence on the nonlinearities of solutions of degenerate parabolic equations. J. Differential Equations 151(2) 231-251

[20] Concha F., Enero 2002, Private communication. 

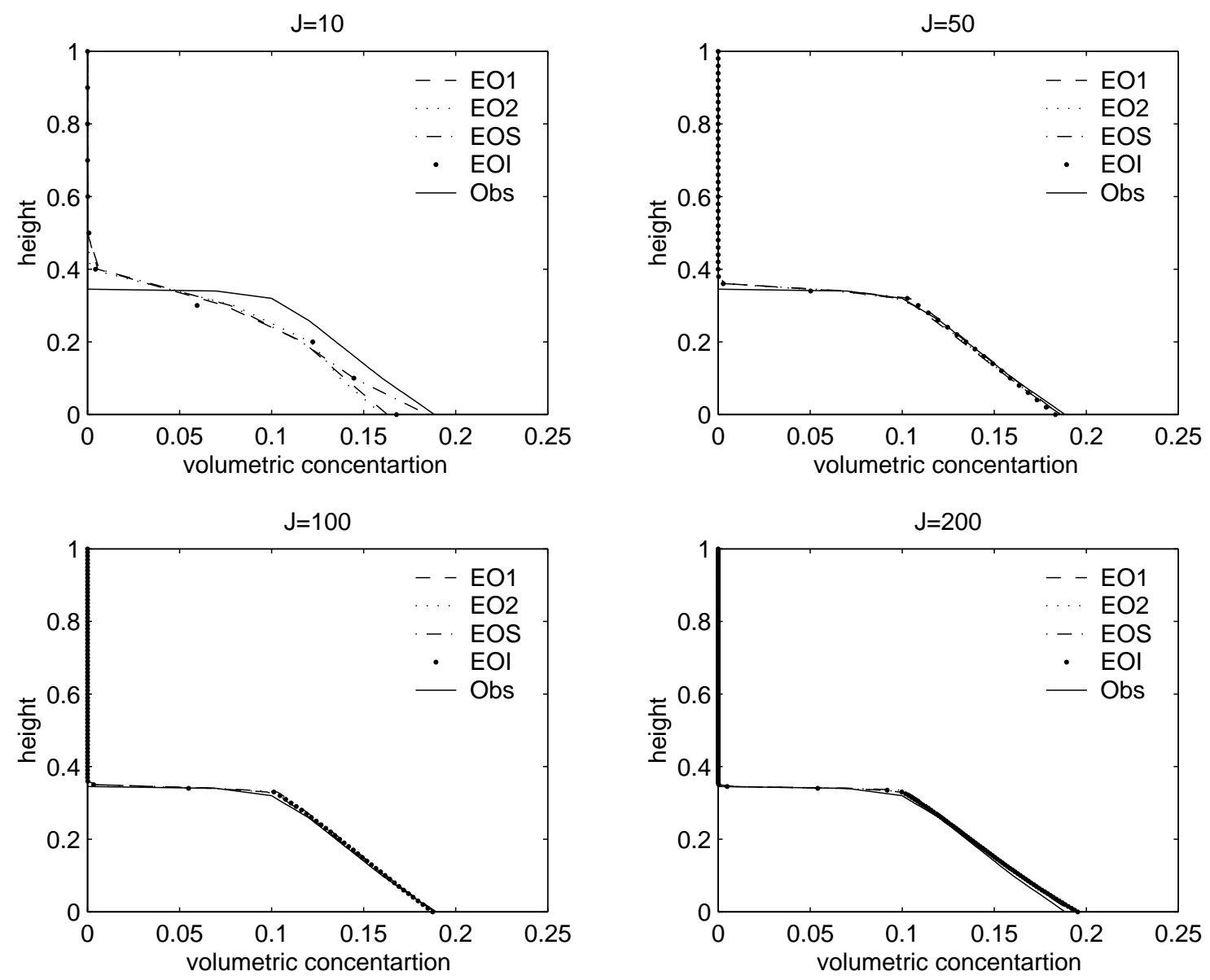

Figure 5. Numerical and observed concentration curves at $T=12144$ for test 2 .

[21] Evje S., Karlsen K. H. and Risebro N. H. 2001 A continuous dependence result for nonlinear degenerate parabolic equations with spatially dependent flux function In H. Freistüler and G. Warnecke, editors, Hyperbolic problems: theory, numerics, applications vol 140 (Birkhäuser Verlag: H. Freistüler and G. Warnecke, editors. Int. Series of Numerical Mathematics) 337-346

[22] Garrido P., Burgos R., Concha F. and Bürger R., Settling velocities of particulate systems: 13. Software for the batch and continuous sedimentation of flocculated suspensions submitted to Int. J. Mineral Process.

[23] Garrido P., Bürger R. and Concha F., 2000 Settling velocities of particulate systems: 11. Comparison of the phenomenological sedimentation-consolidation model with published experimental results Int. J. Mineral Process. 60 213-227.

[24] Gutman S. 1990 Identification of discontinuous parameters in flow equations. SIAM J. Control Optim., 28:1049-1060.

[25] James F. and Sepúlveda M. 1999 Convergence results for the flux identification in a scalar conservation law SIAM J. Control Optim. 37 869-891

[26] James F. and Sepúlveda M. 1994 Parameter identification for a model of chromatographic column Inverse Problems 10 1299-1314

[27] James F. and Sepúlveda M. 1993 Parameter identification for an hyperbolic equation modelling chromatography. Nonlinear Hyperbolic Problems: Theoretical, Applied and Computational Aspects. A. Donato \& F. Oliveri, eds. Notes on Numerical Fluid Mechanics, Vieweg, Wien, 43 347-353.

[28] Karlsen K. H. and Risebro N. H. 2003 On the uniqueness and stability of entropy solutions 


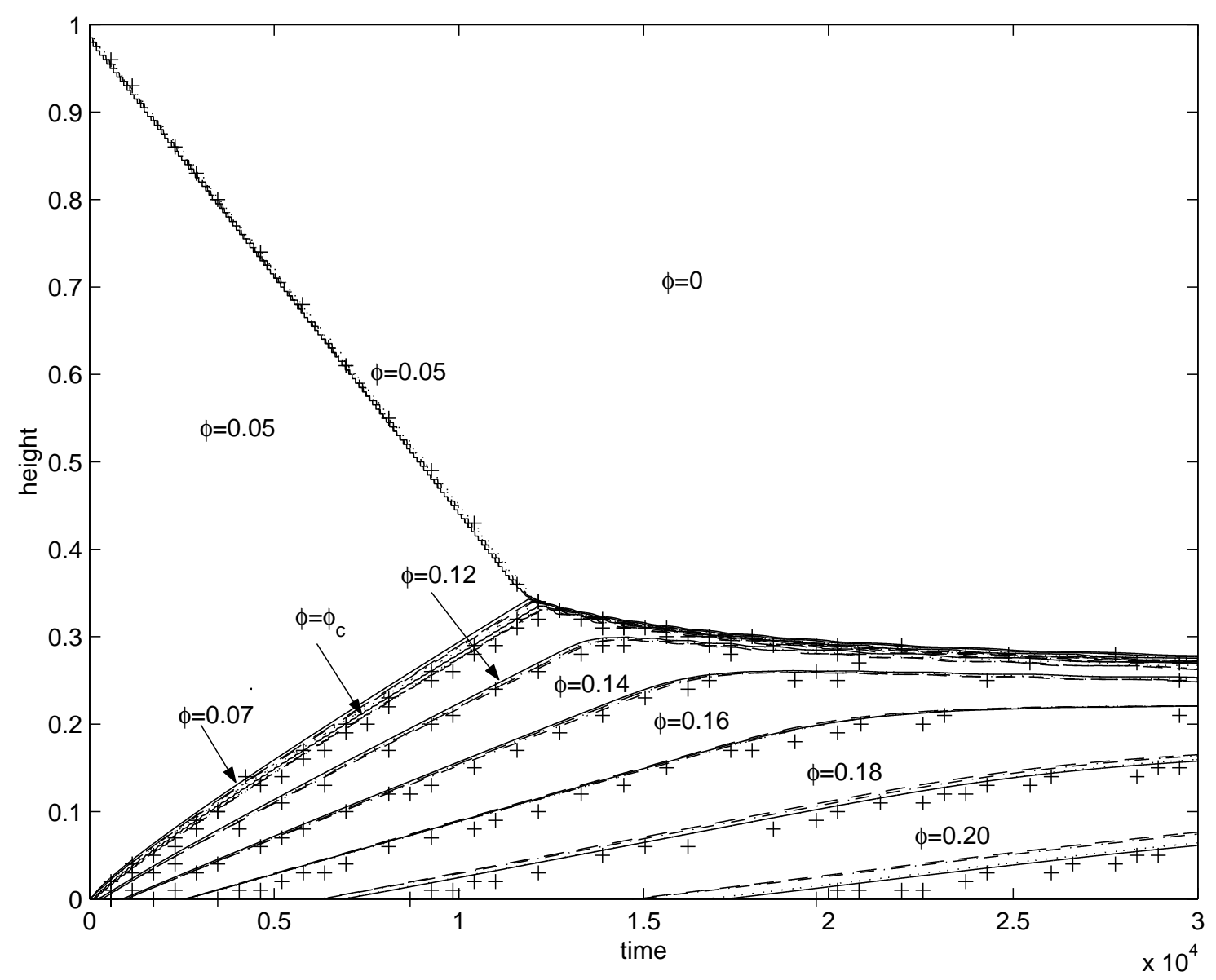

Figure 6. Isoconcentrations curves for test 2 with $M=200, T=30000$. We denote by . - .- the results for EO1, by $\cdots$ for EO2, by - for EOS, by -- for EOI and by +++ for the observation data.

of nonlinear degenerate parabolic equations with rough coefficients Discrete Contin. Dynam. Systems - Series A, 9(5): 1081-1104.

[29] Keung Y.-L. and Zou Y. 1998 Numerical identification of parameters in parabolic systems. Inverse Problems, 14:1299-1314.

[30] Kružkov S. N. 1970 First order quasi-linear equations in several independent variables Math. USSR Sbornik 10(2): 217-243

[31] Kunisch K. and White L. 1985 The parameter estimation problem for parabolic equations and discontinuous observation operators. SIAM J. Control Optim., 23:900-927.

[32] Richardson J. F.and Zaki W. N 1954 The sedimentation of uniform spheres under conditions of viscous flow Chem. Engrg. Science 3 65-73

[33] Yamamoto M. and Zou Y. 2001 Simultaneous reconstruction of the initial temperature and heat radiative coefficient. Inverse Problems, 17:1181-1202. 

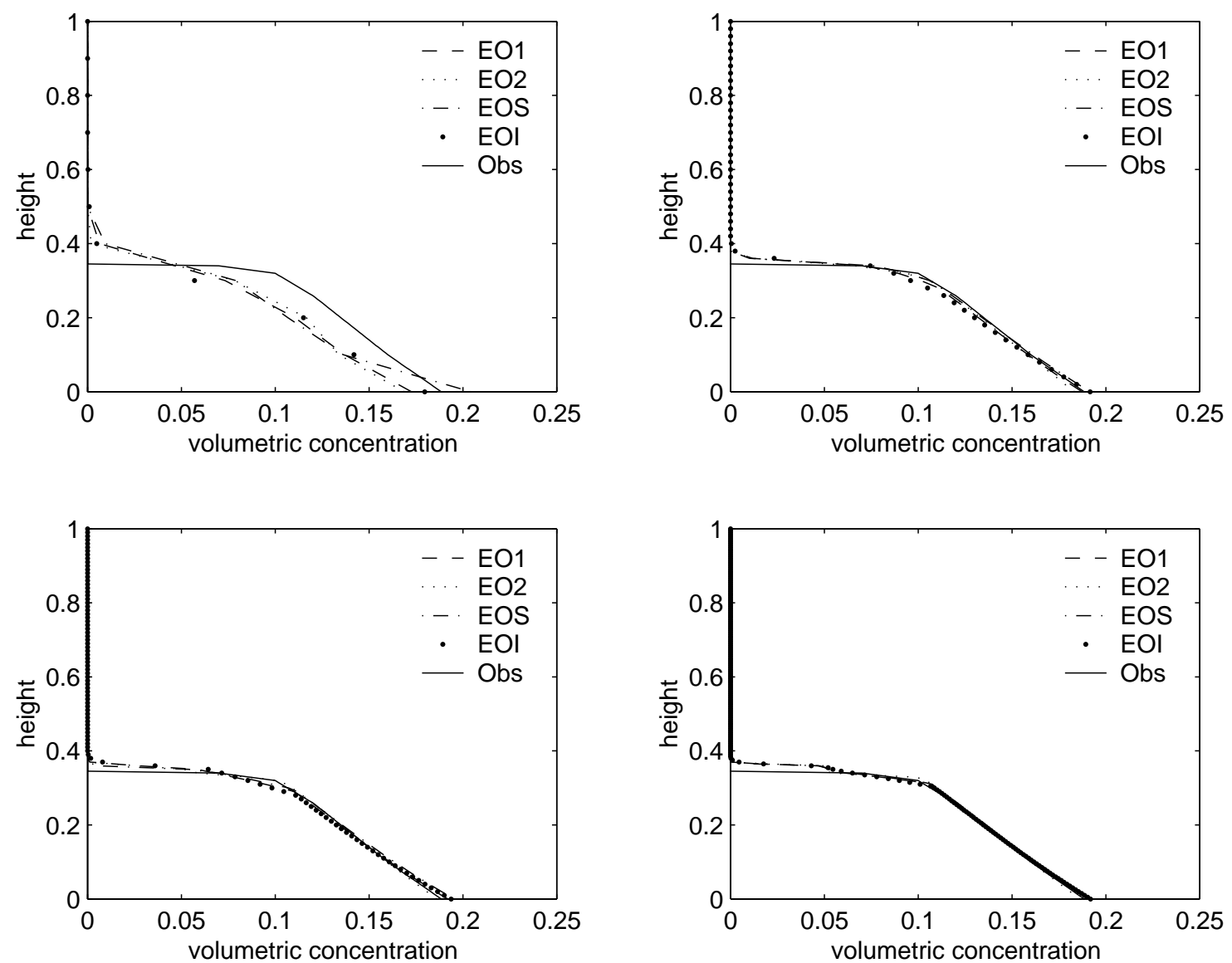

Figure 7. Numerical and observed concentration curves at $T=12144$ for test 3 . 


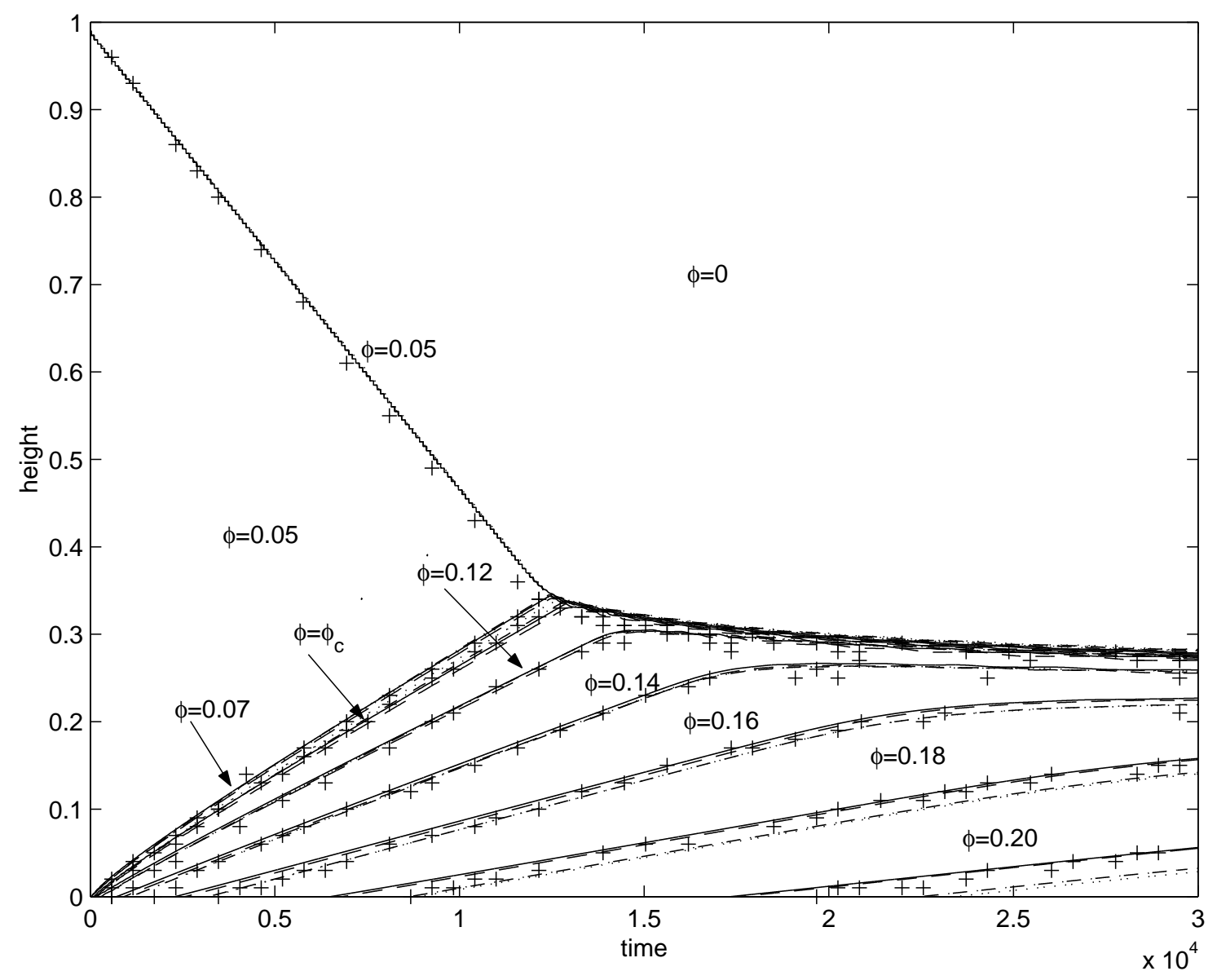

Figure 8. Isoconcentrations curves for test 2 with $M=200, T=30000$. We denote by . - .- the results for EO1, by $\cdots$ for EO2, by - for EOS, by -- for EOI and by +++ for the observation data. 